\title{
Zeeman Effect and g-Values for Neutral Nitrogen and Oxygen
}

\author{
By Carl C. Kiess and George Shortley*
}

\begin{abstract}
Zeeman patterns of red and infrared lines of nitrogen and oxygen, as observed in magnetic fields of 35,000 and 86,000 gausses, at the National Bureau of Standards and the Massachusetts Institute of Technology, respectively, show various degrees of distortion due to Paschen-Back interaction. The interpretation of these patterns has afforded an interesting application of quantum theory to the elucidation of the Paschen-Back effect. The $g$-values derived for the energy levels of $\mathrm{N}$ I and $\mathrm{O}$ I are the first to be announced for neutral atoms of atomic number less than neon, 10, and are found to conform with those required for $L S$-coupling despite the fact that the term intervals do not conform with the Landé ratios.
\end{abstract}

\section{Introduction}

Although various observers have investigated the Zeeman effect of elements in the first two rows of the periodic table, the results, for the most part, are qualitative, most of the published measurements referring apparently to unresolved patterns. It was in the spectra of helium and oxygen that Paschen and Back [1] ${ }^{1}$ first encountered the distorted patterns that appear for close multiplet lines when excited in strong fields. The only Zeeman effects for elements of atomic number below Ne 10 from which $g$-values can be derived are those published by Mihul [2] for $\mathrm{O}$ II, and by Green and Maxwell [3] for $\mathrm{N}$ II.

During the past 10 years, at the National Bureau of Standards, Zeeman-effect observations have been made of the infrared spectra of several metals including chromium, molybdenum, columbium, tantalum, and uranium. On nearly all the spectrograms there are registered the magnetic patterns of the red and infrared lines of atmospheric nitrogen and oxygen, as well as those of the metal lines. Similar spectrograms of molybdenum, manganese, and columbium, secured

\footnotetext{
*Mendenhall Laboratory of Physies, Ohio State University; the analysis reported in this paper was made by Dr. Shortley while employed at the Bureau during the summer of 1947.

1 Figures in brackets indicate the literature references at the end of this paper.
}

with the spectrographs and magnet of the Massachusetts Institute of Technology, have been made available to us by G. R. Harrison. All of these plates have been measured and reduced, and the collected results are presented in this paper.

Because the Zeeman patterns considered here all show various degrees of Paschen-Back interaction, it is necessary to consider this effect in detail in order to derive $g$-values from the measurements. We discuss, therefore, not only the method of obtaining the $g$-values from distorted magnetic patterns, but also the method of computing theoretically the wavelength and intensity shifts arising from Paschen-Back interaction, and give comparisons of the theoretical with the observed results.

\section{Experimental Procedure}

The procedure employed in making the MIT spectrograms has been described frequently by Professor Harrison and collaborators [4]. The National Bureau of Standards observations were obtained with a large, water-cooled Weiss magnet in conjunction with spectrographs in which are mounted concave gratings that were ruled with 15,000 and 30,000 lines per inch by R. W. Wood [5]. These instruments have dispersions of 5.0 



Figure 1. Zeeman patterns of oxygen and nitrogen multiplets.

A bove, the O I quintet 7771 to $7775 \mathrm{~A}$ with fields of (a) 34,600 and (b) 85,400 gausses. Below, the N I quartet 7423 to $7468 \mathrm{~A}$ with a field of 85,400 gausses. Wavelengths increase from left to right.

and $1.8 \mathrm{~A} / \mathrm{mm}$, respectively, in the first-order spectra, whereas that of the MIT spectrograph is $1.6 \mathrm{~A} / \mathrm{mm}$. The light source was a Back [6] lamp in which an interrupted direct-current are was operated on 1 ampere at 220 volts, the air pressure within the arc enclosure being reduced to onesixth atm. The gap between the iron pole-pieces of the magnet was about $6 \mathrm{~mm}$, so that with currents of 160 amp applied to the coils, fields of approximately 35,000 gausses were attained. For calibrating the fields, the spectrograms were exposed also to arcs between other electrodes to secure resolved patterns of known separations.

The National Bureau of Standards gratings are in Wadsworth mountings [7], in which the grating is illuminated by parallel light. Because of the stigmatic properties of a grating mounted in this manner, it was possible to photograph simultaneously both polarizations of the Zeeman pattern, one above the other, by separating them with a Wollaston prism. This prism, of quartz, was placed between the pole-gap of the magnet and the lens that projected the light onto the slit of the spectrograph. A movable occulting bar in front of the slit and between the two polarized images reserved an unexposed strip on the photographic plates for recording the spectrum without the magnetic field. 


\section{Results}

The Zeeman patterns of the $\mathrm{N}$ I lines are presented in tables 1 and 2 . The entries in table 1 are weighted mean values derived from two to five NBS spectrograms for all the lines except the first three, which were measured on only one plate. The nine lines of the table, of wavelength longer than $8,500 \mathrm{~A}$, were measured only on spectrograms with a dispersion of $5 \mathrm{~A} / \mathrm{mm}$ and, therefore, are not so accurately determined as the others. At magnetic field strengths of approximately 35,000 gausses, employed in the NBS observations, no striking anomalies in the spacing or the intensities of the components in the Zeeman patterns are apparent. These patterns, closely resembling those given by Landé's procedure for LS-coupling, are given in the last column of table 1.

On the MIT spectrograms, however, taken at fields in excess of 85,000 gausses, distortions of the patterns both as to spacing and intensity of the components are apparent, even to casual inspection. This is illustrated in figure 1 in the case of the line at 7,442 A. Measurement of the three available MIT spectrograms has yielded the data entered in detail in table 2.

In the first column of table 2 are given the term combinations of the lines, their undisturbed wavelengths according to Edlén [8], and the value in angstrom units of one-half the normal Lorentz triplet corresponding to these wavelengths for the field strengths used. The wavelengths entered in the second column are mean values and probably are in error by a small amount, since there were on the plates no standards between which to make accurate interpolations. On only one of the plates is a no-field exposure available in juxtaposition with both the $\sigma$-and $\pi$-components. In the discussion in section VIII, therefore, each pattern is referred to the arbitrary origin of wavelength listed with its components, since we are concerned only with wavelength differences within a pattern. The third column contains the estimated intensities of the magnetic components. In the last three columns of table 2 are listed the polarizations of the components and the transitions to which they are due, the distances in Lorentz units of the components from their origins, and, finally, their distances from the centers of their patterns. The centers can be located only after the positions of the magnetic levels have been
TABLE 1. Zeeman patterns of $\mathrm{N}_{\mathrm{I}}$ lines from NBS spectrograms.

\begin{tabular}{|c|c|c|}
\hline $\begin{array}{l}\text { Wave- } \\
\text { lengths }\end{array}$ & Term combinations & Observed Zeeman patterns \\
\hline 8718.82 & $3 s{ }^{4} \mathrm{P}_{21 / 2}-3 p{ }^{4} \mathrm{D}_{21 / 2}^{\circ}$ & $(0.57) 1.48$ \\
\hline 8711.69 & $3 s^{4} \mathrm{P}_{11 / 2}-3 p{ }^{4} \mathrm{D}_{11 / 2}^{\circ}$ & $(0.78) 0.89, \mathbf{1 . 5 0}, 2.01$ \\
\hline 8703. 24 & $3 s{ }^{4} \mathrm{P}_{01 / 2}-3 p{ }^{4} \mathrm{D}_{01 / 2}^{\circ}$ & (1.33) 1.33 \\
\hline 8686. 13 & $3 s{ }^{4} \mathrm{P}_{01 / 2}-3 p{ }^{4} \mathrm{D}_{11 / 2}^{\circ}$ & $(0.73) \mathbf{0 . 4 5}, 1.89$ \\
\hline 8683.38 & $3 s{ }^{4} \mathrm{P}_{11 / 2}-3 p{ }^{4} \mathrm{D}_{21 / 2}^{\circ}$ & $(\mathbf{0 . 1 6}, 0.52) \mathbf{0 . 8 3}, 1.17$ \\
\hline 8680.24 & $3 s{ }^{4} \mathrm{P}_{21 / 2}-3 p{ }^{4} \mathrm{D}_{31 / 2}^{\circ}$ & $(0.12 w) 1.03 / \|$ \\
\hline 8629. 24 & $3 s^{2} \mathrm{P}_{11 / 2}-3 p{ }^{2} \mathrm{P}_{11 / 2}^{\circ}$ & $(0.00) 1.32$ \\
\hline 8594.01 & $3 s^{2} \mathrm{P}_{01 / 2}-3 p{ }^{2} \mathrm{P}_{01 / 2}^{\circ}$ & $(0.00) 0.64$ \\
\hline 8567. 74 & $3 s^{2} \mathrm{P}_{01 / 2}-3 p{ }^{2} \mathrm{P}_{11 / 2}^{\circ}$ & $(0.30) 1.05, \mathbf{1 . 6 6}$ \\
\hline 8223. 07 & $3 s^{4} \mathrm{P}_{11 / 2}-3 p{ }^{4} \mathrm{P}_{01 / 2}^{\circ}$ & $(0.47) \quad 1.25,2.20$ \\
\hline 8216. 28 & $3 s{ }^{4} \mathrm{P}_{21 / 2}-3 p{ }^{4} \mathrm{P}_{21 / 2}^{\circ}$ & $(0.00) \quad 1.59$ \\
\hline 8210.64 & $3 s{ }^{4} \mathrm{P}_{11 / 2}-3 p{ }^{4} \mathrm{P}_{11 / 2}^{\circ}$ & $(0.00) \quad 1.73$ \\
\hline 8200.31 & $3 s^{4} \mathrm{P}_{01 / 2}-3 p{ }^{4} \mathrm{P}_{01 / 2}^{\circ}$ & $(0.00) 2.66$ \\
\hline 8187.95 & $3 s{ }^{4} \mathrm{P}_{01 / 2}-3 p{ }^{4} \mathrm{P}_{11 / 2}^{\circ}$ & $(0.47) 1.28,2.20$ \\
\hline 8184. 80 & $3 s^{4} \mathrm{P}_{11 / 2}-3 p{ }^{4} \mathrm{P}_{21 / 2}^{o}$ & $(0.00 w) 1.48 / \Pi$ \\
\hline 7468. 29 & $3 s{ }^{4} \mathrm{P}_{21 / 2}-3 p{ }^{4} \mathrm{~S}_{11 / 2}$ & $\begin{array}{l}(\mathbf{0 . 2 0 , 0 . 6 1 )} \mathbf{1 . 0 1}, 1.42 \\
\quad 1.80,2.19\end{array}$ \\
\hline 7442.28 & $3 s{ }^{4} \mathrm{P}_{11 / 2}-3 p{ }^{4} \mathrm{~S}_{11 / 2}^{\circ}$ & $\begin{array}{l}(0.16,0.39) 1.61,1.88 \\
\quad 2.16\end{array}$ \\
\hline 7423.63 & $3 s{ }^{4} \mathrm{P}_{01 / 2}-3 p{ }^{4} \mathrm{~S}_{11 / 2}$ & $(0.34) \mathbf{1 . 6 8}, 2.35$ \\
\hline
\end{tabular}

fixed according to the procedure discussed below.

In the spectrum of the neutral oxygen atom, two multiplets are easily excited in ares-in-air between metallic electrodes: that at $8,446 \mathrm{~A}$, representing the term combination $2 p^{3} 3 s^{3} \mathrm{~S}^{\circ}-3 p{ }^{3} \mathrm{P}$, and that at 7,771 to $7,775 \mathrm{~A}$ from $2 p^{3} 3 s^{5} \mathrm{~S}^{\circ}-3 p^{5} \mathrm{P}$. In each of these multiplets, the no-field spacing of the three lines is less than the magnetic splitting of the levels, even for moderate fields, so that their Zeeman patterns all exhibit Paschen-Back interaction. In the case of the 8,446 lines, the interaction is essentially complete. In a field of 35,000 gausses the pattern, as measured on a spectrogram taken with the 15,000 lines/inch grating, is $\pm(\mathbf{0 . 0 0} w, 0.99) \mathbf{0 . 9 9}$, with strong $\sigma$-components and very weak $\pi$-components at the separation of the normal Lorentz triplet. The strong, widened $\pi$-component appears as an unresolved group of two or more lines. On an 
TABLE 2. Zeeman patterns of $\mathrm{N}$ I lines from MIT spectrograms

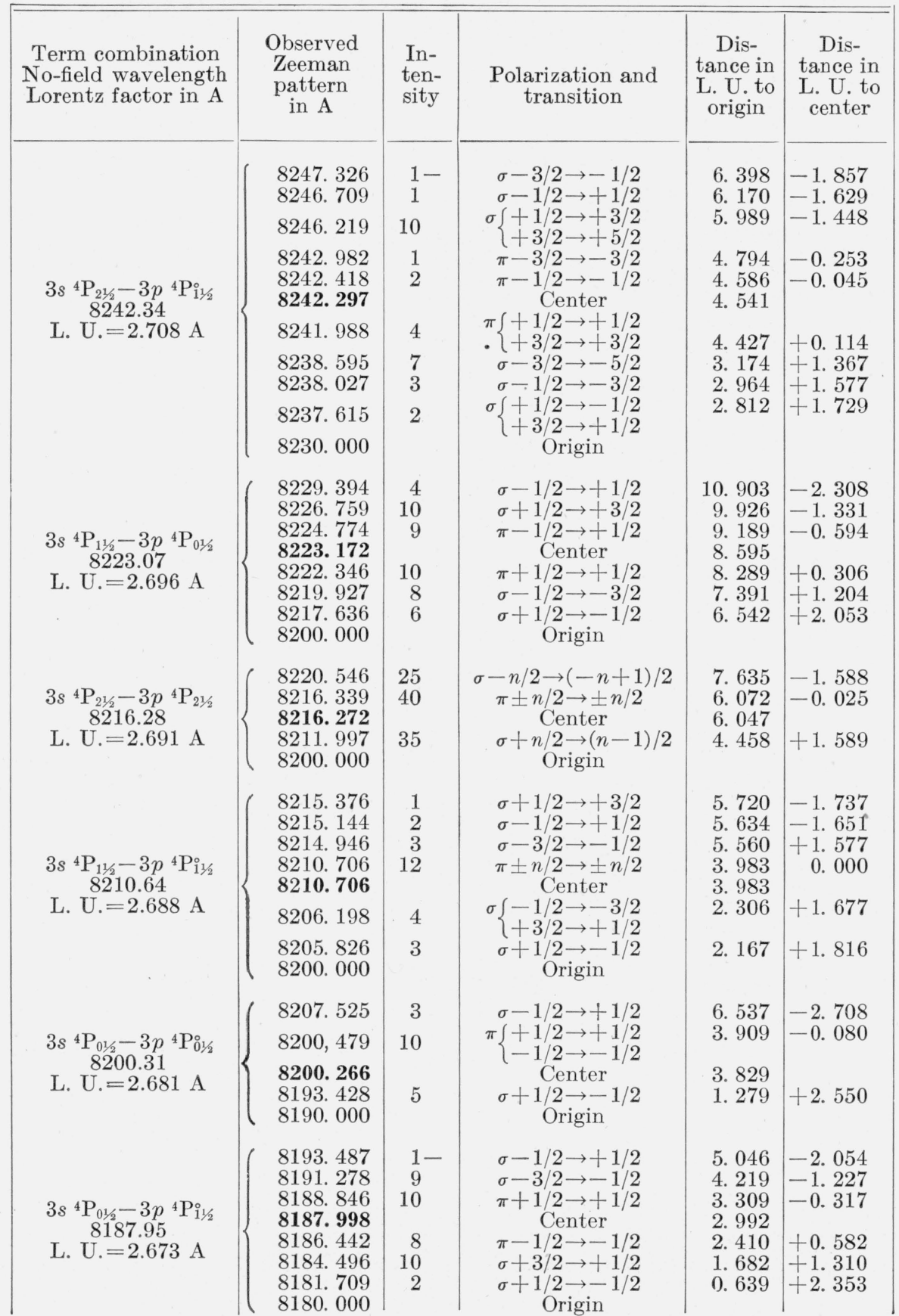


TABLE 2. Zeeman patterns of $\mathrm{N}_{1}$ lines from MIT spectrograms-Continued

\begin{tabular}{|c|c|c|c|c|c|}
\hline $\begin{array}{l}\text { Term combination } \\
\text { No-field wavelength } \\
\text { Lorentz factor in A }\end{array}$ & $\begin{array}{l}\text { Observed } \\
\text { Zeeman } \\
\text { pattern } \\
\text { in A }\end{array}$ & $\begin{array}{l}\text { In- } \\
\text { ten- } \\
\text { sity }\end{array}$ & $\begin{array}{c}\text { Polarization and } \\
\text { transition }\end{array}$ & $\begin{array}{l}\text { Dis- } \\
\text { tance in } \\
\text { L. U. to } \\
\text { origin }\end{array}$ & $\begin{array}{l}\text { Dis- } \\
\text { tance in } \\
\text { L. U. to } \\
\text { center }\end{array}$ \\
\hline $\begin{array}{c}3 s{ }^{4} \mathrm{P}_{11 / 2}-3 p{ }^{4} \mathrm{P}_{21 / 2} \\
8184.80 \\
\text { L. } \mathrm{U} .=2.671 \mathrm{~A}\end{array}$ & $\begin{array}{l}8189.479 \\
8189.317 \\
8188.928 \\
8188.505 \\
8185.274 \\
8185.038 \\
8184.848 \\
8184.660 \\
8184.209 \\
8181.067 \\
8180.763 \\
8180.390 \\
8179.892 \\
8170.000\end{array}$ & $\begin{array}{l}1- \\
1 \\
3 \\
6 \\
4 \\
6 \\
7 \\
5 \\
8 \\
3 \\
2 \\
1-\end{array}$ & $\begin{array}{c}\sigma+1 / 2 \rightarrow+3 / 2 \\
\sigma-1 / 2 \rightarrow+1 / 2 \\
\sigma-3 / 2 \rightarrow-1 / 2 \\
\sigma-5 / 2 \rightarrow-3 / 2 \\
\pi+3 / 2 \rightarrow+3 / 2 \\
\pi+1 / 2 \rightarrow+1 / 2 \\
\text { Center } \\
\pi-1 / 2 \rightarrow-1 / 2 \\
\pi-3 / 2 \rightarrow-3 / 2 \\
\sigma+5 / 2 \rightarrow+3 / 2 \\
\sigma+3 / 2 \rightarrow+1 / 2 \\
\sigma+1 / 2 \rightarrow-1 / 2 \\
\sigma-1 / 2 \rightarrow-3 / 2 \\
\text { Origin }\end{array}$ & $\begin{array}{l}\text { 7. } 293 \\
\text { 7. } 232 \\
\text { 7. } 087 \\
\text { 6. } 928 \\
\text { 5. } 718 \\
\text { 5. } 630 \\
\text { 5. } 559 \\
\text { 5. } 489 \\
\text { 5. } 320 \\
\text { 4. } 143 \\
\text { 4. } 030 \\
\text { 3. } 890 \\
\text { 3. } 704\end{array}$ & $\begin{array}{l}-1.733 \\
-1.673 \\
-1.528 \\
-1.369 \\
-0.159 \\
-0.071 \\
+0.070 \\
+0.239 \\
+1.416 \\
+1.529 \\
+1.669 \\
+1.855\end{array}$ \\
\hline $\begin{array}{c}3 s{ }^{4} \mathrm{P}_{21 / 2}-3 p{ }^{4} \mathrm{~S}_{11 / 2} \\
7468.29 \\
\text { L. U. }=2.224 \mathrm{~A}\end{array}$ & $\begin{array}{l}7473.347 \\
7472.441 \\
7471.484 \\
7470.565 \\
7469.734 \\
7468.870 \\
7468.336 \\
7467.989 \\
7467.075 \\
7466.109 \\
7465.262 \\
7464.396 \\
7463.511 \\
7460.000\end{array}$ & $\begin{array}{r}3 \\
5 \\
6 \\
12 \\
8 \\
12 \\
\\
10 \\
8 \\
12 \\
8 \\
5 \\
2\end{array}$ & $\begin{array}{c}\sigma-3 / 2 \rightarrow-1 / 2 \\
\sigma-1 / 2 \rightarrow+1 / 2 \\
\sigma+1 / 2 \rightarrow+3 / 2 \\
\sigma+3 / 2 \rightarrow+5 / 2 \\
\pi-3 / 2 \rightarrow-3 / 2 \\
\pi-1 / 2 \rightarrow-1 / 2 \\
\text { Center } \\
\pi+1 / 2 \rightarrow+1 / 2 \\
\pi+3 / 2 \rightarrow+3 / 2 \\
\sigma-3 / 2 \rightarrow-5 / 2 \\
\sigma-1 / 2 \rightarrow-3 / 2 \\
\sigma+1 / 2 \rightarrow-1 / 2 \\
\sigma+3 / 2 \rightarrow+1 / 2 \\
\text { Origin }\end{array}$ & $\begin{array}{l}\text { 6. } 001 \\
\text { 5. } 594 \\
\text { 5. } 164 \\
\text { 4. } 750 \\
\text { 4. } 377 \\
\text { 3. } 988 \\
\text { 3. } 748 \\
\text { 3. } 592 \\
\text { 3. } 181 \\
\text { 2. } 747 \\
\text { 2. } 366 \\
\text { 1. } 977 \\
\text { 1. } 579\end{array}$ & $\begin{array}{l}-2.253 \\
-1.846 \\
-1.416 \\
-1.002 \\
-0.629 \\
-0.240 \\
+0.156 \\
+0.567 \\
+1.001 \\
+1.386 \\
+1.771 \\
+2.169\end{array}$ \\
\hline $\begin{array}{c}3 s{ }^{4} \mathrm{P}_{11 / 2}-3 p{ }^{4} \mathrm{~S}_{11 / 2} \\
7442.28 \\
\text { L. U. }=2.208 \mathrm{~A}\end{array}$ & $\begin{array}{l}7447.142 \\
7446.565 \\
7445.840 \\
7443.214 \\
7442.720 \\
\mathbf{7 4 4 2} .378 \\
7442.096 \\
7441.460 \\
7438.790 \\
7438.302 \\
7437.722 \\
7430.000\end{array}$ & $\begin{array}{r}6 \\
10 \\
8 \\
15 \\
2 \\
1- \\
12 \\
5 \\
7 \\
6\end{array}$ & $\begin{array}{c}\sigma-3 / 2 \rightarrow-1 / 2 \\
\sigma-1 / 2 \rightarrow+1 / 2 \\
\sigma+1 / 2 \rightarrow+3 / 2 \\
\pi-3 / 2 \rightarrow-3 / 2 \\
\pi-1 / 2 \rightarrow-1 / 2 \\
\text { Center } \\
\pi+1 / 2 \rightarrow+1 / 2 \\
\pi+3 / 2 \rightarrow+3 / 2 \\
\sigma-1 / 2 \rightarrow-3 / 2 \\
\sigma+1 / 2 \rightarrow-1 / 2 \\
\sigma+3 / 2 \rightarrow+1 / 2 \\
\text { Origin }\end{array}$ & $\begin{array}{l}\text { 7. } 764 \\
\text { 7. } 502 \\
\text { 7. } 174 \\
\text { 5. } 985 \\
\text { 5. } 761 \\
\text { 5. } 606 \\
\text { 5. } 478 \\
\text { 5. } 190 \\
\text { 3. } 981 \\
\text { 3. } 760 \\
\text { 3. } 497\end{array}$ & $\begin{array}{l}-2.158 \\
-1.896 \\
-1.568 \\
-0.379 \\
-0.155 \\
+0.128 \\
+0.416 \\
+1.625 \\
+1.846 \\
+2.109\end{array}$ \\
\hline $\begin{array}{c}3 s^{4} \mathrm{P}_{01 / 2}-3 p{ }^{4} \mathrm{~S}_{1 / 1 / 2} \\
7423.63 \\
\text { L. U. }=2.197_{-}^{-} \mathrm{A}\end{array}$ & $\begin{array}{l}7428.795 \\
7427.324 \\
7424.385 \\
\text { 7423. } 777 \\
7422.911 \\
7419.949 \\
7418.509 \\
7410.000\end{array}$ & $\begin{array}{l}3 \\
6 \\
7 \\
7 \\
7 \\
6 \\
2\end{array}$ & $\begin{array}{c}\sigma-1 / 2 \rightarrow+1 / 2 \\
\sigma-3 / 2 \rightarrow-1 / 2 \\
\pi+1 / 2 \rightarrow+1 / 2 \\
\text { Center } \\
\pi-1 / 2 \rightarrow-1 / 2 \\
\sigma+3 / 2 \rightarrow+1 / 2 \\
\sigma+1 / 2 \rightarrow-1 / 2 \\
\text { Origin }\end{array}$ & $\begin{array}{l}\text { 8. } 555 \\
\text { 7. } 885 \\
\text { 6. } 548 \\
\text { 6. } 271 \\
\text { 5. } 877 \\
\text { 4. } 528 \\
\text { 3. } 873\end{array}$ & $\begin{array}{l}-2.282 \\
-1.614 \\
-0.277 \\
+0.394 \\
+1.743 \\
+2.398\end{array}$ \\
\hline
\end{tabular}


MIT spectrogram, taken at 82,000 gausses, the $\pi$-component and the $\sigma$-component of shorter wavelength are split into two lines of unequal intensities, separated respectively by 0.047 and 0.034 Lorentz unit. The $\sigma$-component of longer wavelength is perceptibly broadened, but unresolved. No faint $\pi$-components appear at the normal triplet position.

The 7,771 to 7,775 multiplet, however, bears no resemblance either to a weak-field pattern or to a Lorentz triplet. The distortions produced by Paschen-Back interaction due to two fields of 34,660 and 85,400 gausses are shown in figure 1 . The data given in table 3 are the wavelengths and estimated intensities of the $\pi$ - and $\sigma$-components and their shifts from the undisturbed positions of the lines in terms of the normal Lorentz triplet spacing. The shifts, in columns (a), (b), and (c) of the table, were calculated from the observed wavelengths by the procedure described in the following section. As with the nitrogen lines, no attempt has been made to express the wavelengths on the correct I. A. scale, because of the absence of standards on the spectrograms. They differ from this by a small constant amount which is of no importance in the interpretation of the pattern. The tabulated wavelengths are mean values derived from measurements of two NBS spectrograms at 34,660 gausses and three MIT spectrograms at 85,400 gausses. For purposes of comparison, the wavelengths from both sets of spectrograms are referred to the same origin at $7771.950 \mathrm{~A}$. The separations $2.220 \mathrm{~A}$ and $1.220 \mathrm{~A}$ adopted for the oxygen lines

TABLE 3. Zeeman pattern of $\mathrm{O}$ I multiplet at $77 \% 1$ to $77 \% 5$ A

\begin{tabular}{|c|c|c|c|c|c|c|c|c|c|}
\hline \multirow{2}{*}{ Wavelength } & \multirow{2}{*}{$\begin{array}{l}\text { Inten- } \\
\text { sity }\end{array}$} & \multicolumn{3}{|c|}{$\begin{array}{l}\text { Lorentz spacing } \\
\quad H=34,660\end{array}$} & \multirow{2}{*}{ Wavelength } & \multirow{2}{*}{$\begin{array}{l}\text { Inten- } \\
\text { sity }\end{array}$} & \multicolumn{3}{|c|}{$\begin{array}{l}\text { Lorentz spacing } \\
\quad H=85,400\end{array}$} \\
\hline & & (a) & (b) & (c) & & & (a) & (b) & (c) \\
\hline \multicolumn{5}{|c|}{$\pi$ components } & \multicolumn{5}{|c|}{$\pi$ components } \\
\hline & & & & & 7769.972 & 3 & -0.821 & & \\
\hline 7771. 230 & 4 & -0.736 & & & 7770.451 & 6 & -0.622 & & \\
\hline 7771.494 & 8 & -0.466 & & & 7770.980 & 7 & -0.403 & & \\
\hline 7771. 784 & 10 & -0.170 & & & 7771.649 & 8 & -0.125 & & \\
\hline 7771.950 & & Origin & & & 7771.950 & & Origin & & \\
\hline 7772.110 & 12 & +0.164 & & & 7772.917 & 13 & +0.401 & & \\
\hline \multirow[t]{2}{*}{7772.486} & 12 & +0.548 & & & 7773.475 & 6 & & -0.288 & \\
\hline & & & & & 7773.579 & 7 & & -0.245 & \\
\hline \multirow[t]{2}{*}{ 7773. 929} & $50 w$ & & -0.247 & & 7773.695 & 10 & & -0.197 & \\
\hline & & & & & 7773. 796 & 7 & & -0.155 & \\
\hline 7774.170 & & & Origin & & 7774.170 & & & Origin & \\
\hline 7774.590 & 15 & & +0.428 & & & & & & \\
\hline 7775.359 & 10 & & & -0.032 & & & & & \\
\hline 7775. 390 & & & & Origin & 7775. 390 & & & & Origin \\
\hline 7775. 764 & 8 & & & +0.382 & 7775.598 & 8 & & +0.593 & \\
\hline \multirow[t]{3}{*}{7776.077} & 4 & & & +0.702 & 7776.354 & 7 & & & +0.400 \\
\hline & & & & & 7776. 904 & 5 & & & +0.629 \\
\hline & & & & & 7777. 376 & 4 & & & +0.824 \\
\hline
\end{tabular}


TABLE 3. Zeeman pattern of $\mathrm{O}$ I multiplet at $7 \% 71$ to $7 \% 75$ A-Continued

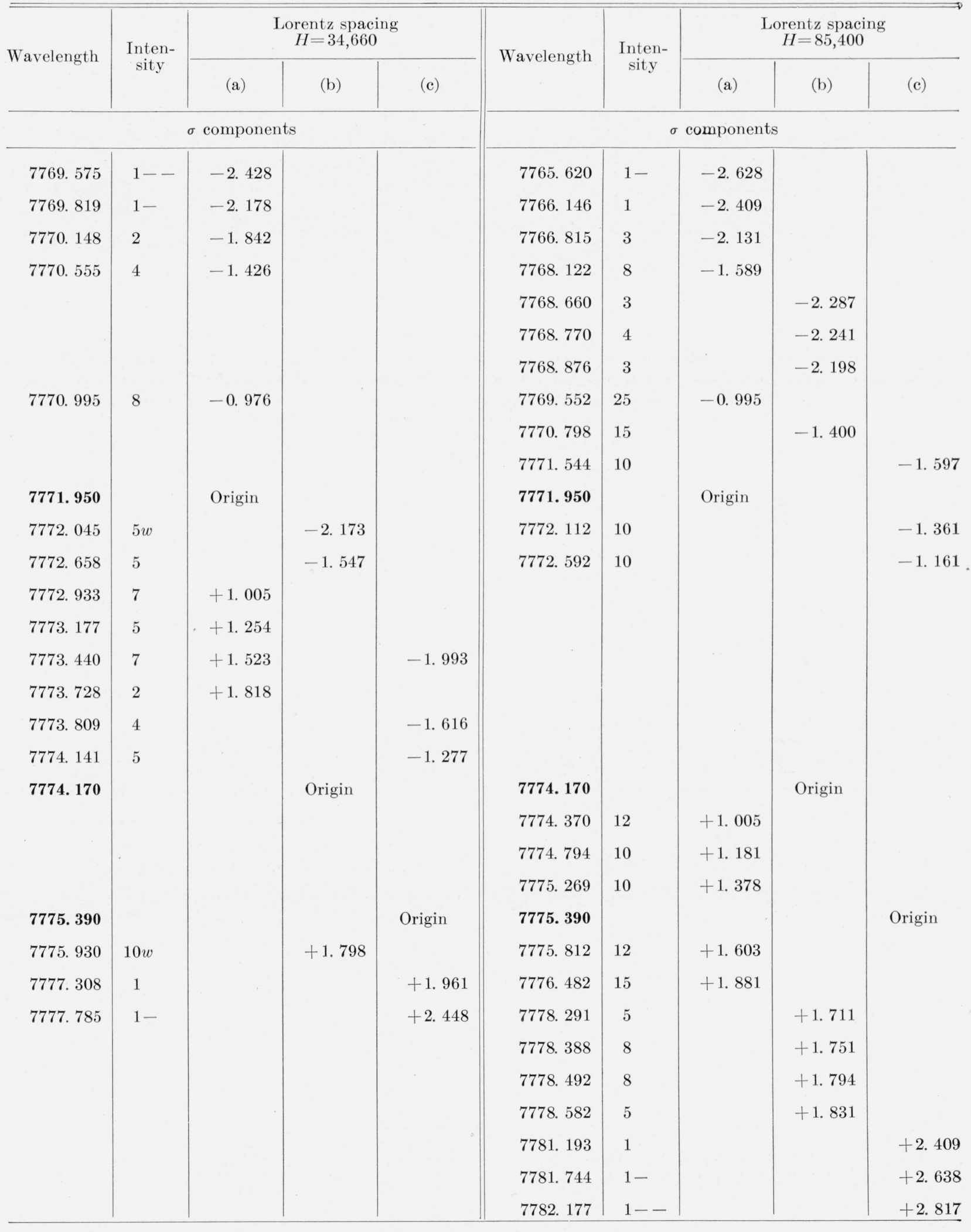


in their no-field positions are mean values from the present series of measurements and from the wavelengths in the solar spectrum as reported by Meggers [9] and by the observers of the Mt. Wilson Observatory [10].

The $g$-values that have been obtained from the data of tables 2 and 3 are given in table 4 . The method used to determine $g$-values of the perturbed terms is discussed in detail in a later section of this paper. The $g$ 's entered in the table for the doublet terms of $\mathrm{N}$ I are only estimated values, which is indicated by enclosing them in parentheses. The three measured doublet patterns do not yield the $g$ 's of the doublet terms uniquely, but they are sufficiently close to the patterns of $L S$-coupling to justify the conclusion that the $g$ 's do not deviate appreciably from the Landé values.

TABLE 4. Observed g-values for energy levels of $\mathrm{N}$ I and $\mathrm{O}$ I

Values in pure $L S$-coupling are given for comparison

\begin{tabular}{|c|c|c|c|c|c|c|c|}
\hline \multicolumn{4}{|c|}{$\mathrm{N}$ I } & \multicolumn{4}{|c|}{$\mathrm{O}_{\mathrm{I}}$} \\
\hline Term & $J$ & $g_{o b s}$ & $g_{L S}$ & Term & $J$ & $g_{o b s}$ & $g_{L S}$ \\
\hline $3 s{ }^{4} \mathrm{P}$ & $\left\{\begin{array}{l}21 / 2 \\
11 / 2\end{array}\right.$ & $\begin{array}{l}\text { 1. } 603 \\
\text { 1. } 735\end{array}$ & $\begin{array}{l}\text { 1. } 600 \\
\text { 1. } 733\end{array}$ & & 2 & 1. $999 \pm 0.002$ & \\
\hline $3 s^{2} \mathrm{P}$ & $\left\{\begin{array}{l}01 / 2 \\
1 \frac{1}{2} \\
01 / 2\end{array}\right.$ & $\begin{array}{l}2.670 \\
(1.33) \\
(0.67)\end{array}$ & $\begin{array}{l}\text { 2. } 667 \\
\text { 1. } 33 \\
0.67\end{array}$ & $3 p^{5} \mathrm{P}$ & $\begin{array}{l}3 \\
2 \\
1\end{array}$ & $\begin{array}{l}\text { 1. } 666 \pm 0.001 \\
\text { 1. } 836 \pm 0.003 \\
\text { 2. } 506 \pm 0.005\end{array}$ & $\begin{array}{l}\text { 1. } 667 \\
\text { 1. } 833 \\
\text { 2. } 500\end{array}$ \\
\hline $3 p{ }^{4} \mathrm{D}^{\circ}$ & $\left\{\begin{array}{l}31 / 2 \\
21 / 2 \\
11 / 2 \\
01 / 2\end{array}\right.$ & $\begin{array}{l}\text { 1. } 44 \\
\text { 1. } 36 \\
\text { 1. } 19 \\
0.002\end{array}$ & $\begin{array}{l}\text { 1. } 43 \\
1.37 \\
1.20 \\
0.00\end{array}$ & & & & \\
\hline $3 p^{4} \mathrm{P}^{\circ}$ & $\left\{\begin{array}{l}21 / 2 \\
11 / 2 \\
01 / 2\end{array}\right.$ & $\begin{array}{l}\text { 1. } 598 \\
\text { 1. } 737 \\
\text { 2. } 671\end{array}$ & $\begin{array}{l}\text { 1. } 600 \\
\text { 1. } 733 \\
\text { 2. } 667\end{array}$ & & & & \\
\hline $3 p{ }^{4} \mathrm{~S}^{\circ}$ & $11 / 2$ & 2. 004 & 2. 000 & & & & \\
\hline $3 p^{2} \mathrm{P}^{\circ}$ & $\left\{\begin{array}{l}1 \frac{1}{2} \\
01 / 2\end{array}\right.$ & $\begin{array}{l}(1.33) \\
(0.67)\end{array}$ & $\begin{array}{l}\text { 1. } 33 \\
0.67\end{array}$ & & & & \\
\hline
\end{tabular}

\section{The ${ }^{5} \mathrm{P} \rightarrow{ }^{5} \mathrm{~S}$ Multiplet of $\mathrm{O}$ I}

The three lines of the Or multiplet $2 p^{3} 3 p{ }^{5} \mathrm{P} \rightarrow$ $3 s^{5} \mathrm{~S}^{\circ}$ are so close that large Paschen-Back perturbations occur with any field large enough to resolve the Zeeman components. The patterns at 34,660 and 85,400 gausses are shown in figure 1 . These patterns are so distorted by Paschen-Back perturbations that they bear little resemblance to weak-field patterns.

The correct assignment of components to states was first determined for the 85,400-gauss pattern by making an entirely theoretical computation of component positions and (strengths) ) $^{1 / 2}$ by using the observed no-field positions of the levels and assuming strict Russell-Saunders coupling in determining the $g$-values and the matrix components that give the Paschen-Back interaction. The method of making these computations will be discussed in detail in sections VI and VII. The computed values are compared with the observed wavelengths and estimated intensities in table 5 and figure 2. Although this pattern is wide for a Zeeman pattern, yet it covers a sufficiently small range of wavelength so that within the observational error it is appropriate to assume that wavenumber differences are proportional to wavelength differences, and use a mean value (at 7,773 A) of the ratio of $\Delta \nu$ to $\Delta \lambda$ to determine the Lorentz splitting factor in angstroms. Because wave-number and wavelength differences have opposite signs, the energy-state pattern of table 5 seems upside down. The scale has been turned over in plotting figure 3 , although angstrom units are retained.

The agreement between the computed and observed wavelength intervals and intensities in 
table 5 and figure 2 is excellent. Apart from the clustered, or very weak components bracketed in table 5 , the maximum wavelength discrepancy is $0.026 \mathrm{~A}$; the average of the discrepancies, all counted positive, is only $0.013 \mathrm{~A}$. This agreement shows that the coupling must be exceedingly close to Russell-Saunders. This fact is of considerable theoretical interest since the ${ }^{5} \mathrm{P}$ term does not obey the Landé interval rule, the intervals being in the ratio $3: 1.65$ in place of $3: 2$. Since the quan- tum numbers of the term must be quite accurately those of ${ }^{5} \mathrm{P}$, the departure from the interval rule is undoubtedly to be accounted for by some type of magnetic interaction other than the ordinary spin-orbit.

The fact that visual estimates of intensity correspond quite accurately to the square roots of the line strengths rather than to the strengths themselves was first pointed out by Russell [11]. There are certain interesting discrepancies in this inten-

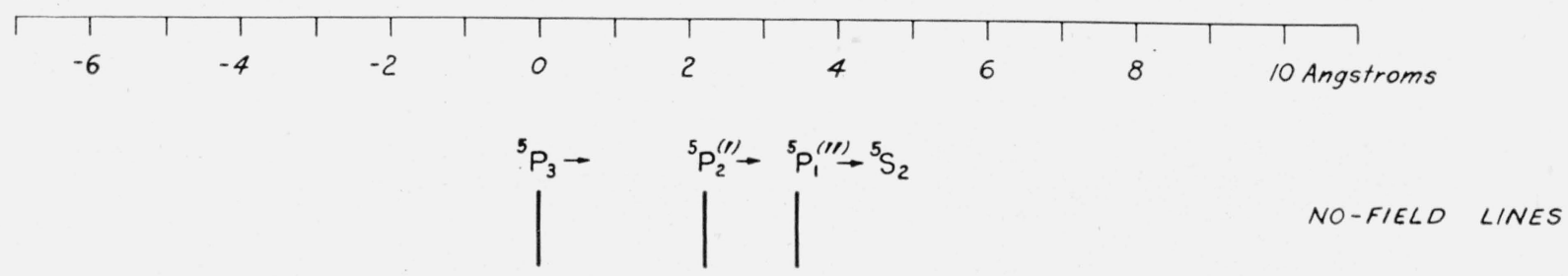
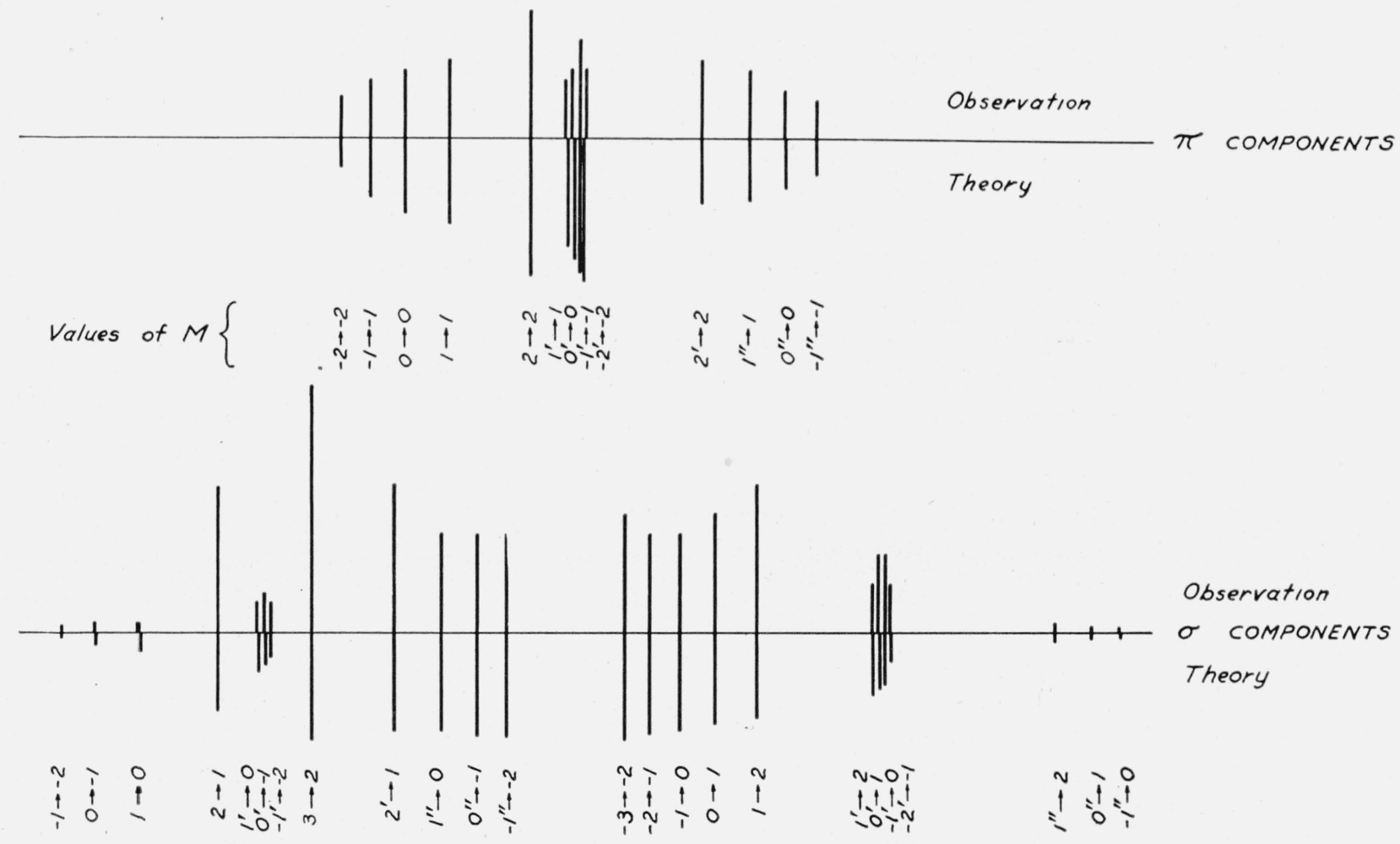

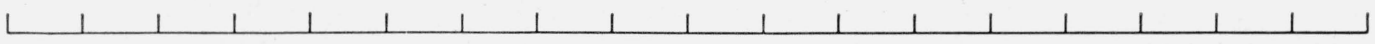

Figure 2. Zeeman patterns of the $\mathrm{O}$ I miltiplet $2 p^{3} 3 p^{5} \mathrm{P} \rightarrow 3 s^{5} \mathrm{~S}$ in a field of 85,400 gausses

Comparison of observation and theory. The theoretical positions were computed by using the no-field positions observed on the same plate and strict LS-coupling matrix components. The lengths of the bars show computed relative values of the square roots of the component strengths below and visual intensity estimates above. 


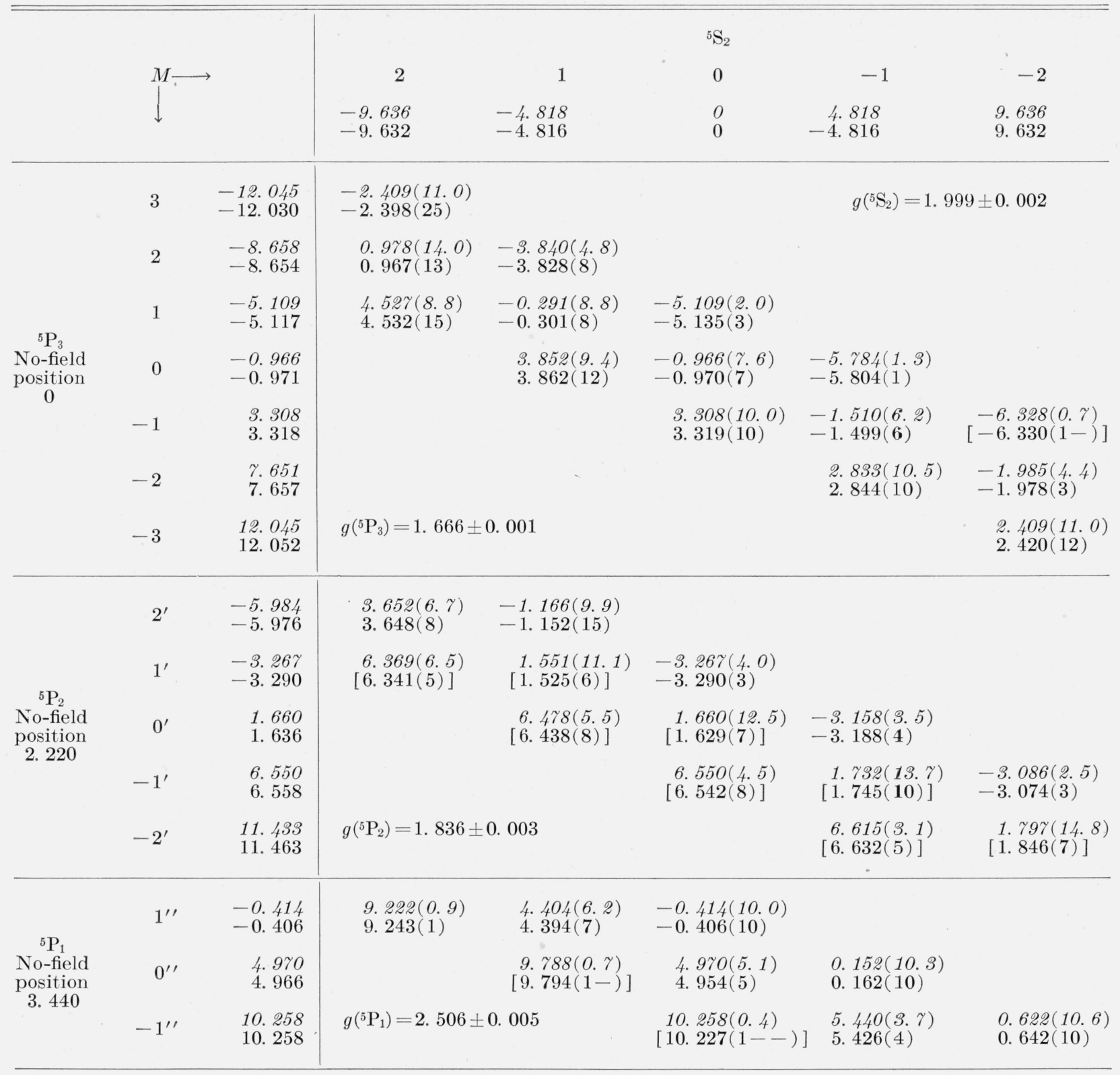

sity comparison that seem to be a definite indication of nonisotropic excitation conditions. In particular, the $\sigma$-component $M=3 \rightarrow 2$ is observed much too strong, and in fact definitely much stronger than $M=-3 \rightarrow-2$. Since these two particular components suffer neither shift in position nor alteration in strength because of PaschenBack interaction, they must remain of equal strength at any value of the field if the excitation is isotropic. They are of substantially equal strength on the NBS plates, but are strikingly unequal on the MIT plates, as can be seen even from the reproduction in figure 1 .

With the components allocated to their proper boxes in table 5 , a state analysis was made and $g$-values computed from the experimental data. Because the pattern is very wide, these $g$-values can be obtained with high accuracy, and are found to agree with the Russell-Saunders values ( 2 for ${ }^{5} \mathrm{~S}_{2}, 5 / 3=1.667$ for ${ }^{5} \mathrm{P}_{3}, 11 / 6=1.833$ for ${ }^{5} \mathrm{P}_{2}$, 2.5 for ${ }^{5} \mathrm{P}_{1}$ ) within the estimated experimental error given in the tables. The method of obtain- 
ing these $g$-values from the state analysis is given in the next section. The entries in table 5 printed in Roman type are the observed components and the energy states derived therefrom, measured in A from the no-field position of ${ }^{5} \mathrm{P}_{3} \rightarrow{ }^{5} \mathrm{~S}_{2}$, with the estimated intensities in parentheses. The bracketed components are poorly resolved or are very weak. The observed Lorentz splitting factor is $2.409 \mathrm{~A}$. The entries in italics are the components, states, and square roots of the line strengths computed from the no-field positions of the lines, from the observed splitting factors, and from the $L S$-coupling $g$-values.

At the right of figure 3 is shown a comparison of the observed positions of the ${ }^{5} \mathrm{P}$ states, as given in table 5, with their unperturbed positions. By unperturbed position, we mean the position the state would have if there were no Paschen-Back perturbation by states belonging to neighboring levels. The unperturbed position is obtained by adding to the no-field position the quantity $\mathrm{Mg}$ times the Lorenz splitting factor. The difference between the observed position of the state (solid bar in figure 3 ) and the unperturbed position (broken bar) is the Paschen-Back perturbation.

It is only states of the same $M$-value that perturb each other. Theory shows rigorously that the sum of the Paschen-Back perturbations of the states of the same $M$-value must vanish. Thus in figure 3 there is only one state of $M=3$ and one of $M=-3$, so these are unperturbed. The two states of $M=2$ and the two of $M=-2$ should be shifted away from each other by equal amounts. The sum of the three perturbations of the three states of $M=1$, of $M=0$, and of $M=-1$ should be zero in each case. The data given on figure 3 agree with these statements within the accuracy with which the values of the positions can be obtained from the experimental data used.

\section{Analysis of a Paschen-Back Pattern}

Usually it is not necessary to make a theoretical computation in order to allocate properly the components of a well-resolved Paschen-Back pattern. In fact, such a theoretical computation is impossible unless the $g$-values and the type of coupling are already fairly well known. ${ }^{2} \quad$ Instead one looks

\footnotetext{
2 The theory of the Paschen-Back effect in intermediate coupling has been discussed by J. B. Green and J. F. Eichelberger, Phys. Rev. 56, 51 (1939).
}

for repeated differences, always between a $\pi$ component and a $\sigma$-component, and attempts to make a state-analysis of the components in the same way as an ordinary level-analysis is made of spectral lines, by making use of all available theoretical clues with regard to Paschen-Back perturbations and intensities. Table 6, which shows the same oxygen quintet as table 5 , but at a much lower field, will serve as an example, although a difficult one because of unresolved components. The numbers in the table printed in Roman type are the observed wavelengths of the components decreased by 7,700, and, in parentheses, their estimated intensities. The differences of the $\pi$ and $\sigma$ components that correspond to the ${ }^{5} \mathrm{~S}_{2}$ splitting are entered between the columns, the bracketed values being uncertain, owing to blending or weakness of the lines. Here the observed Lorentz splitting factor is $0.978 \mathrm{~A}$. In italics are given the states and components computed from the differences between the states. The states for the blends in ${ }^{5} \mathrm{P}_{2}$ are in all cases placed so that the mean Paschen-Back perturbation is zero, as required by theory.

The analysis in table 6 is simplified by the fact that the lower term ${ }^{5} \mathrm{~S}_{2}^{\circ}$ is unperturbed, since it contains only one level. Hence the interval between the states of ${ }^{5} \mathrm{~S}_{2}^{\circ}$ occurs repeatedly, each $\pi$-component having a $\sigma$-component at this distance on one or both sides, according to its position in the table. This interval is easily picked up (average $1.947 \mathrm{~A}$ ), and determines the $g$-value of ${ }^{5} \mathrm{~S}_{2}^{\circ}$. There are, however, two fairly strong $\sigma$-components $(M=3 \rightarrow 2,-3 \rightarrow-2)$ that do not lie at this interval from any $\pi$-component. Furthermore, these two $\sigma$-components are equidistant from the no-field position of ${ }^{5} \mathrm{P}_{3} \rightarrow{ }^{5} \mathrm{~S}_{2}^{\circ}$, which makes them easy to spot. In this way the $M=3,-3$ separation and the $g$-value of ${ }^{5} \mathrm{P}_{3}$ are determined, and the unperturbed positions of the other states of ${ }^{5} \mathrm{P}_{3}$ may be computed. The table is completed by using the following guides:

(a) Intensities. For a discussion of the effect of Paschen-Back perturbations on intensity patterns, see sections VII and VIII.

(b) The fact that the sum of the Paschen-Back perturbations on states of a given $M$-value vanishes. Since the $+M$ and $-M$ unperturbed states are equidistant from the no-field position, this guide is best used in connection with the mean 


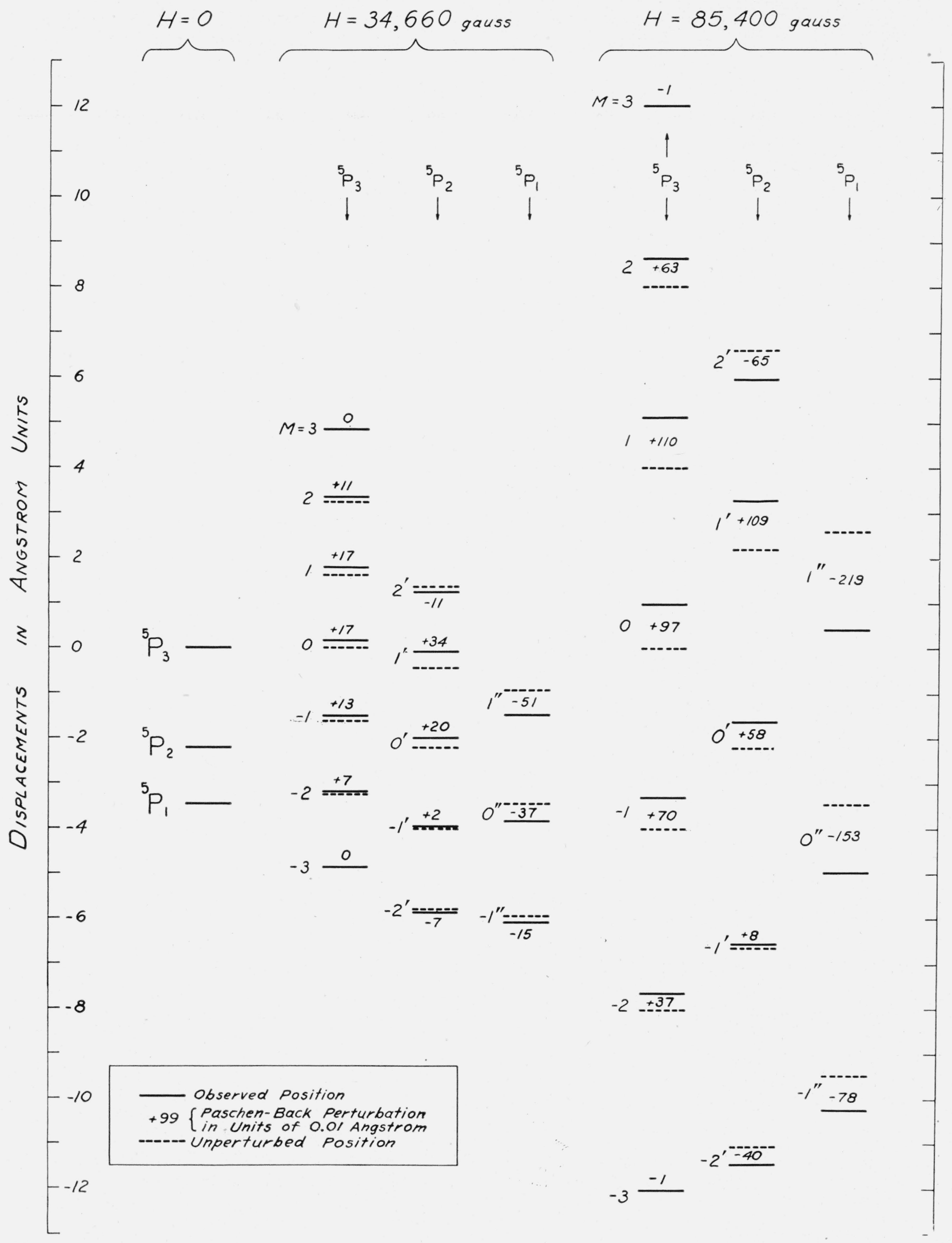


positions of perturbed states of opposite $M$-value. Thus, the mean of the states $M=3$ and $M=-3$ is the no-field position of ${ }^{5} \mathrm{P}_{3}$; the mean of the four states, $M=2,-2,2^{\prime},-2^{\prime}$ is the mean of the no-field positions of ${ }^{5} \mathrm{P}_{3}$ and ${ }^{5} \mathrm{P}_{2}$, etc. (fig. 3 ). Thus, if we denote the mean positions of a set of states or no-field levels by \langle\rangle , we have for the ${ }^{5} \mathrm{P}$ of our example:

$$
\begin{array}{ll}
\langle 3,-3\rangle & ={ }^{5} \mathrm{P}_{3} \\
\left\langle 2,-2,2^{\prime},-2^{\prime}\right\rangle & =\left\langle{ }^{5} \mathrm{P}_{3},{ }^{5} \mathrm{P}_{2}\right\rangle \\
\left\langle 1,-1,1^{\prime},-1^{\prime}, 1^{\prime \prime},-1^{\prime \prime}\right\rangle & =\left\langle{ }^{5} \mathrm{P}_{3},{ }^{5} \mathrm{P}_{2},{ }^{5} \mathrm{P}_{1}\right\rangle \\
\left\langle 0, \quad 0^{\prime}, 0^{\prime \prime}\right\rangle & =\left\langle{ }^{5} \mathrm{P}_{3},{ }^{5} \mathrm{P}_{2},{ }^{5} \mathrm{P}_{1}\right\rangle
\end{array}
$$

(c) When $g$-values are finally determined and unperturbed positions computed, the perturbations must be in the right direction and of the right order of magnitude. Thus, looking at the $H=$ 34,600 -gauss part of figure 3 , which shows the perturbations in the example in question, the single states of $M=3$ and $M=-3$ are unperturbed. The two states of $M=2$ and the two of $M=-2$ push each other apart by equal amounts, but the unperturbed states of $M=2$ are closer than those of $M=-2$, so the $M=2$ states suffer av greater perturbation. The perturbations are approximately in inverse ratio to the unperturbed separations. The three states of $M=1$ are more closely grouped than those of $M=-1$ so they suffer greater perturbations. In each case the top state must be pushed higher and the bottom state lower. The center state is pushed up more strongly by the closer state of the same $M$ below it than it is pushed down by the more distant state above, so it suffers a net upward perturbation. The statement of this last sentence is not a rigorous requirement, because the matrix components determining the magnitude of the perturbation may, for example, be different for $\left(1,1^{\prime}\right)$ and $\left(1^{\prime}\right.$, $\left.1^{\prime \prime}\right)$. The states of $M=0$ behave similarly to those of $M= \pm 1$.

\section{Figure 3. Paschen-Back perturbations of the $\mathrm{O}_{\mathrm{I}} 2 p^{3} 3 p^{5} \mathrm{P}$ states at two field strengths.}

Solid bars show observed state positions as obtained from an empirical state analysis. The unperturbed positions shown by broken bars were computed by using the observed no-field positions and the empirical $g$-values obtained from the state analysis.
Determination of g-values. When a satisfactory state analysis is completed, the computation of the $g$-values, which give the weak field, or unperturbed, state positions, is straightforward. We can use the observed data in table 5, which give the best values for the oxygen quintet, as an illustration.

The ${ }^{5} \mathrm{~S}_{2}$ splitting is $4.816 \pm 0.004 \mathrm{~A}$, determined from the $12 \pi-\sigma$ intervals between unbracketed components. This splitting, divided by the Lorentz splitting factor, $2.409 \mathrm{~A}$, gives $g\left({ }^{5} \mathrm{~S}_{2}\right)=$ $1.999 \pm 0.002$.

The probable errors that we give here are determined purely from internal consistency of the 12 $\pi-\sigma$ intervals, a possible error in the well-determined Lorentz splitting factor being neglected. On this basis a probable error of $0.010 \mathrm{~A}$ is assigned to each measured (unbracketed) wavelength, and from this the probable errors in the ${ }^{5} \mathrm{P}$ levels and the $g\left({ }^{5} \mathrm{P}\right)$ are determined in straightforward fashion.

The $M=3,-3$ interval of $24.082 \pm 0.018 \mathrm{~A}$, divided by 6 gives $4.014 \pm 0.003 \mathrm{~A}$ for the unperturbed ${ }^{5} \mathrm{P}_{3}$ splitting, and $g\left({ }^{5} \mathrm{P}_{3}\right)=1.666 \pm 0.001$.

The observed $(2,-2)$ and $\left(2^{\prime},-2^{\prime}\right)$ intervals are distorted by Paschen-Back perturbations, but because of the properties of these perturbations it is seen that the sum of these two intervals is the same as if there were no perturbation and can be used for deriving $g\left({ }^{5} \mathrm{P}_{2}\right)$. The sum of these two intervals is $33.750 \pm 0.018 \mathrm{~A}$. Divided by 4 , this gives $8.438 \pm 0.005 \mathrm{~A}$ for the sum of the unperturbed ${ }^{5} \mathrm{P}_{3}$ and ${ }^{5} \mathrm{P}_{2}$ splittings and $8.438-4.014=$ $4.424 \pm 0.006 \mathrm{~A}$ for the ${ }^{5} \mathrm{P}_{2}$ splitting, corresponding to $g\left({ }^{5} \mathrm{P}_{2}\right)=1.836 \pm 0.003$.

Similarly, the sum of the three observed inter$\operatorname{vals}(1,-1),\left(1^{\prime},-1^{\prime}\right),\left(1^{\prime \prime},-1^{\prime \prime}\right)$ must be the same as the sum of the unperturbed intervals. The sum of the observed intervals is $28.947 \pm 0.022 \mathrm{~A}$. Divided by 2 , this gives $14.474 \pm 0.011 \mathrm{~A}$ for the sum of the unperturbed ${ }^{5} \mathrm{P}_{3},{ }^{5} \mathrm{P}_{2},{ }^{5} \mathrm{P}_{1}$ splittings, or $14.474-8.438=6.036 \pm 0.012 \quad \mathrm{~A}$ for the ${ }^{5} \mathrm{P}_{1}$ splitting, corresponding to $g\left({ }^{5} \mathrm{P}_{1}\right)=2.506 \pm 0.005$.

The $g$-values for the narrower pattern of table 6 were determined in exactly the same way but are less accurate, because the states of ${ }^{5} \mathrm{P}_{2}$ belonging to the blended lines are determined only roughly by the line positions and were actually placed as follows: $M=-2^{\prime}$ was placed so that the average of $2,-2,2^{\prime},-2^{\prime}$ coincided with the average of the no-field positions of ${ }^{5} \mathrm{P}_{3}$ and ${ }^{5} \mathrm{P}_{2} . \quad M=0$ was placed 
$\bar{\varnothing}$

TABle 6. Paschen-Back pattern of $\mathrm{O}$ I $2 p^{3} 3 p^{5} \mathrm{P} \rightarrow 3 s^{5} \mathrm{~S}$ (7771 to 7775 A) at 34,660 gausses

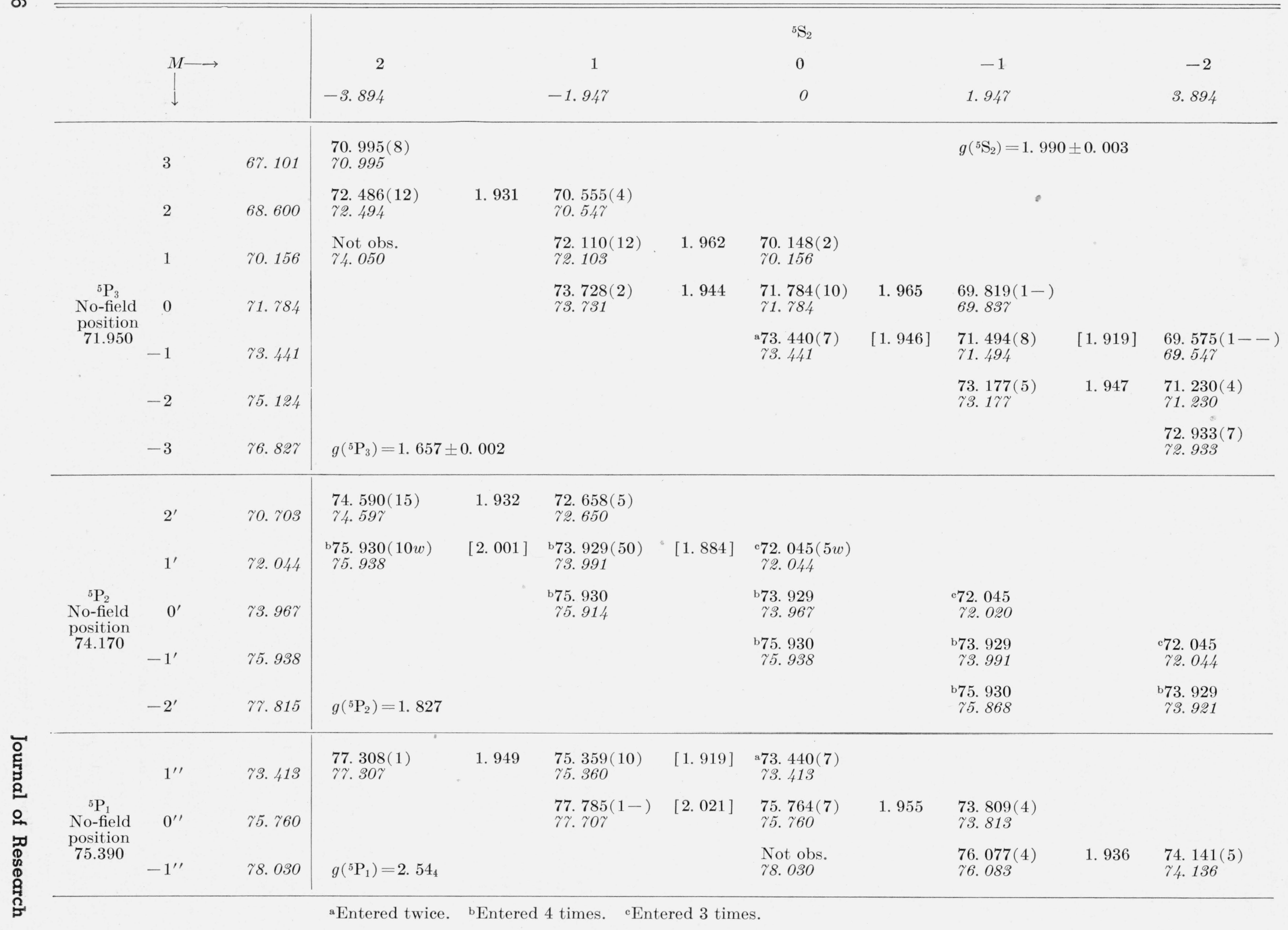


so that the average of $0,0^{\prime}, 0^{\prime \prime}$ coincided with the average no-field position of the three levels. $M=1^{\prime}$ and $M=-1^{\prime}$ were placed so that the average of the six states of $M= \pm 1$ coincided with the average of the no-field positions of the three levels and so that the states $M=1^{\prime}$ and $M=-1^{\prime}$ would give components of equal wavelength. This placement is in satisfactory agreement with observation but is undetermined to within the widths of the blends. Hence $g\left({ }^{5} \mathrm{P}_{2}\right)$, derived from the \pm 1 intervals, is particularly subject to error

The probable errors given for $g\left({ }^{5} \mathrm{~S}_{2}\right)$ and $g\left({ }^{5} \mathrm{P}_{3}\right)$ in table 6 were determined in the same way as those in table 5, any error in the determination of the Lorentz unit being neglected. It will be noted that these two $g$-values in table 6 are smaller than those in table 5, and also smaller than the $L S$ values, by about 1 part in 200 , which is outside of the probable errors given. In table 4 we adopt the values of table 5 from the MIT plates, because the Lorentz unit for the MIT plates is obtained from standard lines photographed simultaneously with the oxygen and nitrogen lines, whereas the NBS Lorentz unit is obtained from standard patterns photographed on the plate in a subsequent exposure. The NBS technique possibly introduces a slight error in the Lorentz unit because of slightly different field strength or different placement of the are in the magnetic field when the standard lines are exposed.

\section{Theoretical Computation of Paschen- Back Patterns in Russell-Saunders Coupling}

The method of computing theoretical PaschenBack patterns in Russell-Saunders coupling is discussed in general terms by Condon and Shortley [12] and elsewhere. An attempt will be made here to give formulas and method, and sufficient detail on the oxygen ${ }^{5} \mathrm{P}$ example, to enable a spectroscopist unfamiliar with details of quantummechanical theory to make such computations, which are really quite straightforward. We shall plunge directly into the example of $\mathrm{O}_{\mathrm{I}}{ }^{5} \mathrm{P}(7,773 \mathrm{~A})$ at 85,400 gauss, giving general formulas as need for their use arises.

In the first place, it is most convenient to use as unit the normal Lorentz splitting, which gives half the width of a normal triplet. This Lorentz unit, in $\mathrm{cm}^{-1}$, is $4.670 \times 10^{-5}$ times $H$ in gausses [13]. It is usually determined directly from known patterns on each exposure. The Lorentz unit in our case is experimentally determined as $2.409 \mathrm{~A}$ at $7,773 \mathrm{~A}$. From the observed no-field wavelength differences, $2.220 \mathrm{~A}$ and $3.440 \mathrm{~A}$ measured from ${ }^{5} \mathrm{P}_{3} \rightarrow{ }^{5} \mathrm{~S}_{2}$, we compute the no-field quintet energies in Lorentz units as ${ }^{5} \mathrm{P}_{3}=0,{ }^{5} \mathrm{P}_{2}=-0.922,{ }^{5} \mathrm{P}_{1}=-1.428$ L. U., placing them in the right order of energies to correspond to the erect quintet with least $J$ value lowest. As our starting point we compute the unperturbed pattern of states, which would obtain if there were no Paschen-Back perturbation, by adding the various values of $M g$ to the above energies, with $g\left({ }^{5} \mathrm{P}_{3}\right)=1.667, g\left({ }^{5} \mathrm{P}_{2}\right)=1.833$, $g\left({ }^{5} \mathrm{P}_{1}\right)=2.500$. Using a superscript to denote the value of $M$, we find for the unperturbed energies:

$$
\begin{aligned}
{ }^{5} \mathrm{P}_{3}^{3} & =3 \times 1.667=5 \mathrm{~L} . \mathrm{U} . \\
{ }^{5} \mathrm{P}_{3}^{2} & =2 \times 1.667=3.333 \\
{ }^{5} \mathrm{P}_{3}^{1} & =1.667 \\
{ }^{5} \mathrm{P}_{3}^{0} & =0 \\
{ }^{5} \mathrm{P}_{3}^{-1} & =-1.667 \\
{ }^{5} \mathrm{P}_{3}^{-2} & =-2 \times 1.667=-3.333 \\
{ }^{5} \mathrm{P}_{3}^{-3} & =-3 \times 1.667=-5 \\
{ }^{5} \mathrm{P}_{2}^{2} & =-0.922+2 \times 1.833=2.745 \\
{ }^{5} \mathrm{P}_{2}^{1} & =-0.922+1.833=0.911 \\
{ }^{5} \mathrm{P}_{2}^{0} & =-0.922 \\
{ }^{5} \mathrm{P}_{2}^{-1} & =-0.922-1.833=-2.755 \\
{ }^{5} \mathrm{P}_{2}^{-2} & =-0.922-2 \times 1.833=-4.589 \\
{ }^{5} \mathrm{P}_{1}^{1} & =-1.428+2.500=1.072 \\
{ }^{5} \mathrm{P}_{1}^{0} & =-1.428 \\
{ }^{5} \mathrm{P}_{1}^{-1} & =-1.428-2.500=-3.928 \mathrm{~L} . \mathrm{U}
\end{aligned}
$$

These values, converted to angstroms by multiplication with 2.409 , correspond to the broken bars on the right of figure 3 .

We now need the nondiagonal matrix elements that determine the Paschen-Back interaction. These elements connect only states of the same $M$, the same $L$ and $S$ (i. e., states of the same term), and having $J$ values differing by one unit. In Lorentz units, the interaction element connecting 
the state $S L J M$ and the state $S L, J-1, M$ is

$\sqrt{\frac{(J-L+S)(J+L-S)(J+L+S+1)(L+S+1-J)}{4 J^{2}(2 J-1)(2 J+1)}} \sqrt{J^{2}-M^{2}}$,

and is always positive. Substitution in this formula of $S=2, L=1, J=3$ gives the interaction elements connecting ${ }^{5} \mathrm{P}_{3}$ and ${ }^{5} \mathrm{P}_{2}$ :

$$
\begin{aligned}
M=+2, M & =-2: & \sqrt{2} / 3 & =0.471 \mathrm{~L} . \mathrm{U} . \\
M=+1, M & =-1: & 4 / 3 \sqrt{5} & =0.596 \mathrm{~L} . \mathrm{U} . \\
M & =\quad 0: & \sqrt{2 / 5} & =0.632 \mathrm{~L} . \mathrm{U} .
\end{aligned}
$$

Substitution of $S=2, L=1, J=2$ gives the elements connecting ${ }^{5} \mathrm{P}_{2}$ and ${ }^{5} \mathrm{P}_{1}$ :

$$
\begin{array}{rr}
M=+1, M=-1: & 3 / 2 \sqrt{5}=0.671 \text { L. U. } \\
M=0 & \sqrt{3 / 5}=0.775 \text { L. U. }
\end{array}
$$

Having computed the unperturbed states and the interaction elements, we proceed with the computation of the perturbed states as follows:

When there is just one state of given $M$, such as $M= \pm 3$, there is no perturbation, and the above unperturbed energies, converted to $\mathrm{A}$, are entered in italics on the left of table 5 with sign changed.

When there are two states of the same $M$, such as $M=2$, let $A$ and $B$ be the upper and lower of the two unperturbed energy values ( $A=3.333$ for ${ }^{5} \mathrm{P}_{3}^{2}, B=2.745$ for ${ }^{5} \mathrm{P}_{2}^{2}$ in our case); let $C$ be the interaction element ( $C=0.471$ from above). Then the energies of the perturbed states are given by the general formula

$$
\frac{1}{2} A+\frac{1}{2} B \pm \sqrt{\left(\frac{1}{2} A-\frac{1}{2} B\right)^{2}+C^{2}} .
$$

The higher of these two values goes with the state associated with the higher of the no-field states (noncrossing rule discussed below). Thus, in our case, with the notation of table 5 and figures 3 and 4 , the perturbed energies for $M=2$ are

$$
\left.\begin{array}{l}
2 \\
2^{\prime}
\end{array}\right\}=3.039 \pm 0.555=\left\{\begin{array}{l}
3.594 \text { L. U. }=8.658 \mathrm{~A} \\
2.484 \text { L. U. }=5.984 \mathrm{~A}
\end{array}\right.
$$

which are the values entered (with sign change) in italics at the left of table 5, and which correspond to the observed energies of figures 3 and 4 .
When there are three or more states of the same $M$, the calculation is more complicated. As an illustration take the case of $M=1$. Write down the matrix

\begin{tabular}{l||lll}
${ }^{5} \mathrm{P}_{3}^{1}$ & 1.667 & 0.596 & 0 \\
${ }^{5} \mathrm{P}_{2}^{1}$ & 0.596 & 0.911 & 0.671 \\
${ }^{5} \mathrm{P}_{1}^{1}$ & & 0.671 & 1.072
\end{tabular}

in which the unperturbed energies are entered along the diagonal, the interaction elements each twice in the appropriate spaces.

From this, form the determinantal equation (the secular equation)

$$
\begin{array}{lll}
1.667-\lambda & 0.596 & 0 \\
0.596 & 0.911-\lambda & 0.671 \\
0 & 0.671 & 1.072-\lambda
\end{array} \mid=0 .
$$

When the determinant is expanded this equation becomes

$$
-\lambda^{3}+3.650 \lambda^{2}-3.477 \lambda+0.497=0 .
$$

The three roots of this equation are the energies of the three perturbed states. In order of decreasing energy these should be designated as $1,1^{\prime}, 1^{\prime \prime}$, associated with ${ }^{5} \mathrm{P}_{3},{ }^{5} \mathrm{P}_{2},{ }^{5} \mathrm{P}_{1}$, respectively. This association should be made in the same order as the no-field energies, not necessarily the order of the unperturbed energies. In this case, as the field is increased from 0 to 85,400 gausses, two of the unperturbed states of $M=1$ cross as shown in figure 4 , so that they are no longer in the order of no-field energies; but if the perturbed energies were computed as a function of field strength, it would be found that states of the same $M$ would never cross (see fine lines in fig. 4), so that the order of association of the perturbed states is to be the same as the no-field order. The three roots of eq 6 give the energies in Lorentz units:

$$
\left.\begin{array}{c}
1=2.121 \text { L. U. }=5.109 \mathrm{~A}, \\
1^{\prime}=1.356 \text { L. U. }=3.267 \mathrm{~A}, \\
1^{\prime \prime}=0.172 \text { L. U. }=0.414 \mathrm{~A} .
\end{array}\right\}
$$

These states are entered in table 5 with reversed sign, and correspond to the observed values of 


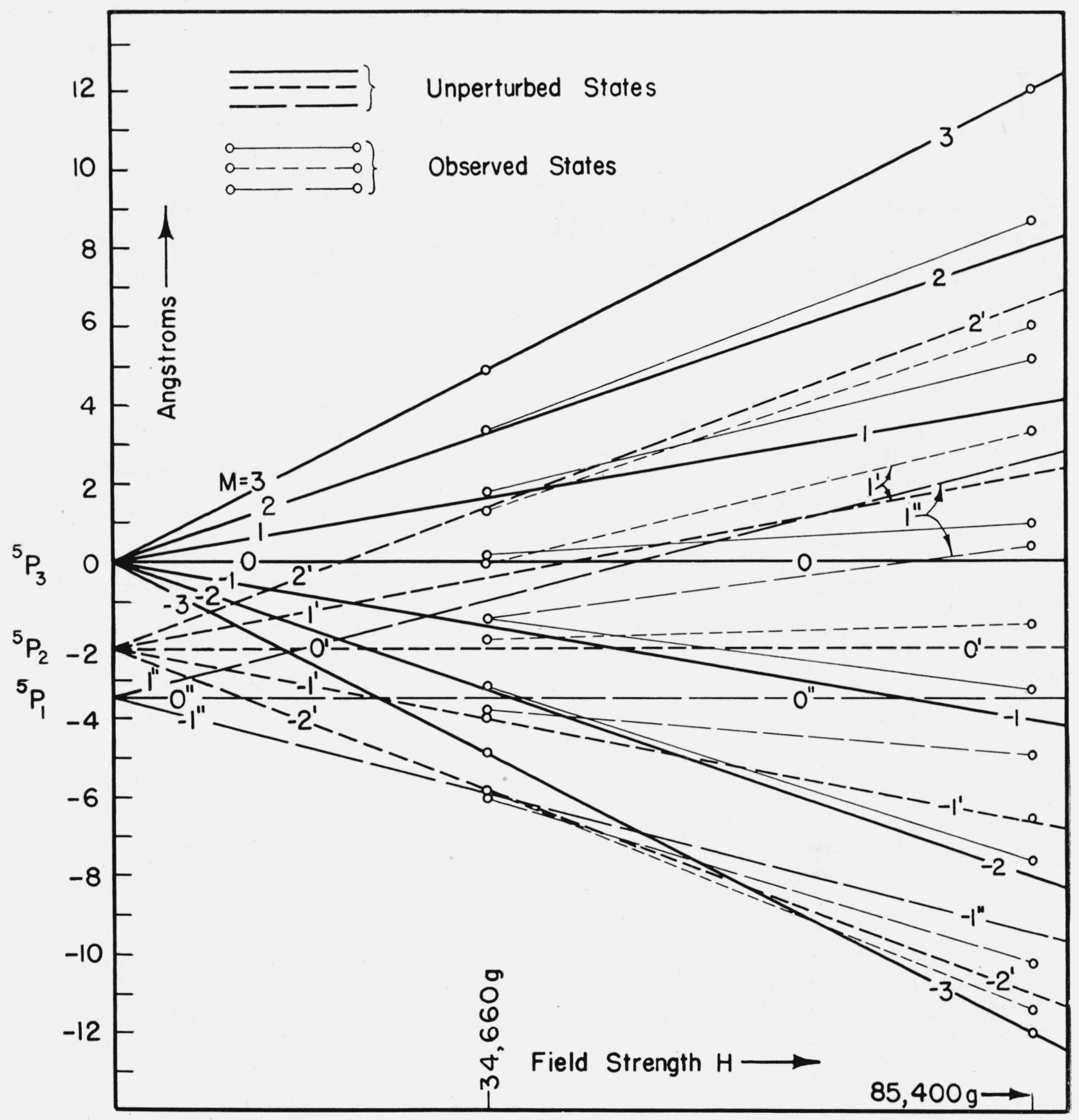

FiguRE 4.-Zeeman effect of the $\mathrm{O}$ I $2 p^{3} 3 p^{5} \mathrm{P}$ tє $m$.

This figure shows (heavy lines) the positions the states would occupy if there were no Paschen-Back perturbation and illustrates, by the light lines connecting two observed points, the growth of the Paschen-Back perturbation with field-strength. Intermediate between the observed points, the actual state positions would, of course, lie on curved lines rather than on the straight lines drawn here. Because the $g$-values of the levels increase in going from ${ }^{5} \mathrm{P}_{3}$ to ${ }^{6} \mathrm{P}_{2}$ to ${ }^{5} \mathrm{P}_{1}$, the unperturbed states of the same positive $M$-value lie closer than those of the corresponding negative $M$-value and are more strongly perturbed. The crossing of the unperturbed states of $M=1$ and the noncrossing of the perturbed states is schematically illustrated, 
figures 3 and 4 . The states for $M=0$ and -1 are obtained by exactly similar computations. The equation corresponding to eq 6 for $M=0$ is

$$
-\lambda^{3}-2.350 \lambda^{2}-0.317 \lambda+0.570=0,
$$

and that for $M=-1$ is

$$
-\lambda^{3}-8.350 \lambda^{2}-21.157 \lambda-15.895=0 .
$$

The same procedure as that above will permit the computation of the Paschen-Back pattern of any term in $L S$-coupling.
VII. Theoretical Computation of Line Strengths in Paschen-Back Patterns in LS-Coupling

In quantum mechanics the strength of a line is defined [12, p. 98] as a quantity that must be multiplied by the fourth power of the frequency and by the number of atoms in any one of the initial states in order to obtain the radiated energy. Since all of the lines of our Paschen-Back pattern have closely the same frequency, we can compare relative strengths directly with observed

\begin{tabular}{|c|c|c|c|c|c|c|c|}
\hline & $\begin{array}{l}M- \\
\downarrow\end{array}$ & \multicolumn{2}{|l|}{2} & 1 & $\begin{array}{c}{ }^{5} \mathrm{~S}_{2} \\
0\end{array}$ & -1 & -2 \\
\hline \multirow{7}{*}{${ }^{5} \mathrm{P}_{3}$} & 3 & $-\sqrt{120}=$ & -10.95 & & & & \\
\hline & 2 & $\sqrt{80}=$ & 8. 94 & $-\sqrt{80}=-8.94$ & & & \\
\hline & 1 & $\sqrt{8}=$ & 2. 83 & $\sqrt{128}=11.31$ & $-\sqrt{48}=-6.93$ & & \\
\hline & 0 & & & $\sqrt{24}=4.90$ & $\sqrt{144}=12.00$ & $-\sqrt{24}=-4.90$ & \\
\hline & -1 & & & & $\sqrt{48}=6.93$ & $\sqrt{128}=11.31$ & $-\sqrt{8}=-2.83$ \\
\hline & -2 & & & & & $\sqrt{80}=8.94$ & $\sqrt{80}=\quad 8.94$ \\
\hline & -3 & & & & & & $\sqrt{120}=10.95$ \\
\hline & $2^{\prime}$ & $\sqrt{160}=$ & 12. 65 & $\sqrt{40}=6.32$ & & & \\
\hline & $1^{\prime}$ & $\sqrt{40}=$ & 6. 32 & $\sqrt{40}=6.32$ & $\sqrt{60}=7.75$ & & . \\
\hline${ }^{5} \mathrm{P}_{2}$ & $0^{\prime}$ & & & $\sqrt{60}=7.75$ & 0 & $\sqrt{60}=7.75$ & \\
\hline & $-1^{\prime}$ & & & & $\sqrt{60}=7.75$ & $-\sqrt{40}=-6.32$ & $\sqrt{40}=$ \\
\hline & $-2^{\prime}$ & & & & & $\sqrt{40}=6.32$ & $-\sqrt{160}=-12.65$ \\
\hline & $1^{\prime \prime}$ & $\sqrt{72}=$ & 8. 49 & $-\sqrt{72}=-8.49$ & $-\sqrt{12}=-3.46$ & & \\
\hline${ }^{5} \mathrm{P}_{1}$ & 0 & & & $\sqrt{36}=6.00$ & $-\sqrt{96}=-9.80$ & $-\sqrt{36}=-6.00$ & \\
\hline & $-1^{\prime \prime}$ & & & & $\sqrt{12}=3.46$ & $-\sqrt{72}=-8.49$ & $-\sqrt{72}=-8.49$ \\
\hline
\end{tabular}

TABLE 7.-Relative values of $\boldsymbol{S}^{3 / 4}$ for components of an unperturbed ${ }^{5} \mathrm{P} \rightarrow{ }^{5} \mathrm{~S}$ multiplet in transverse observation 
relative intensities under the assumption that all the close-lying upper states have the same excitation. The strength of a multiplet is defined as the sum of the strengths of its lines, and the strength of a line as the sum of the strengths of its components.

In making a transformation from one type of coupling to another, such as the transformation from the unperturbed states to the perturbed states in a Paschen-Back pattern (the perturbation mixes up states of different $J$ ), we must work not with the strength itself but with its square root, which we denote by $\boldsymbol{S}^{1 / 2}$. This turns out to be quite convenient in our case, because visual intensity estimates give numbers that are actually close to the square roots of the relative strengths. It is to be noted that $\boldsymbol{S}^{1 / 2}$ is a signed quantity and that it is very important to use the proper sign.

We need first the relative $\boldsymbol{S}^{1 / 2}$ for the lines of a multiplet in $L S$-coupling. These are obtained by taking the square roots of the strengths as given by the familiar Kronig, Sommerfeld-Hönl, Russell formulas [12, p. 238], with the sign given below:

Sign of $S^{1 / 2}(S, L, J ; \quad S, L+\Delta L, J+\Delta J)$ :
$\Delta L=0, \quad \Delta J=0\left\{\begin{array}{l}+ \text { if } S(S+1)<L(L+1)+J(J+1) \\ - \text { if } S(S+1)>L(L+1)+J(J+1)\end{array}\right.$

$\Delta L=0, \quad \Delta J= \pm 1(-)$

$\Delta L= \pm 1, \Delta J=0(+)$

$\Delta L= \pm 1, \Delta J= \pm 1(+)$

$\Delta L= \pm 1, \Delta J=\mp 1(-)$

In our case of ${ }^{5} \mathrm{P}_{3,2,1} \rightarrow{ }^{5} \mathrm{~S}_{2}$, the strengths are in the ratio $7: 5: 3$, and from the above sign rule we obtain the relative values

$$
\begin{array}{r}
\boldsymbol{S}^{1 / 2}\left({ }^{5} \mathrm{P}_{3},{ }^{5} \mathrm{~S}_{2}\right)=+\sqrt{7} ; \quad \boldsymbol{S}^{1 / 2}\left({ }^{5} \mathrm{P}_{2},{ }^{5} \mathrm{~S}_{2}\right)=+\sqrt{5} ; \\
\boldsymbol{S}^{1 / 2}\left({ }^{5} \mathrm{P}_{1},{ }^{5} \mathrm{~S}_{2}\right)=-\sqrt{3} .
\end{array}
$$

We now need the formulas for the strengths of the unperturbed Zeeman components of a line in terms of the whole line strength, which is denoted by $\boldsymbol{S}(J, J+\Delta J)$ in the formulas below, since these formulas are good for any line whether the coupling is Russell-Saunders or not. Per unit solid angle at angle $\theta(0 \leqq \theta \leqq \pi)$ with the magnetic field, these strengths are

$$
\left.\begin{array}{l}
\boldsymbol{S}_{\theta}^{1 / 2}(J, M ; J+1, M)=2 \boldsymbol{S}^{1 / 2}(J, J+1) \sqrt{\frac{3}{32 \pi} \frac{(J+1)^{2}-M^{2}}{(J+1)(2 J+1)(2 J+3)}} \sin \theta \\
\boldsymbol{S}_{\theta}^{1 / 2}(J, M ; J+1, M \pm 1)=\mp \boldsymbol{S}^{1 / 2}(J, J+1) \sqrt{\frac{3}{32 \pi} \frac{(J \pm M+1)(J \pm M+2)}{(J+1)(2 J+1)(2 J+3)}} \sqrt{1+\cos ^{2} \theta} \\
\boldsymbol{S}_{\theta}^{1 / 2}(J, M ; J, M)=2 M \boldsymbol{S}^{1 / 2}(J, J) \sqrt{\frac{3}{32 \pi} \frac{1}{J(J+1)(2 J+1)}} \sin \theta \\
\boldsymbol{S}_{\theta}^{1 / 2}(J, M ; J, M \pm 1)=\boldsymbol{S}^{1 / 2}(J, J) \sqrt{\frac{3}{32 \pi} \frac{(J \mp M)(J \pm M+1)}{J(J+1)(2 J+1)}} \sqrt{1+\cos ^{2} \theta} \\
\boldsymbol{S}_{\theta}^{1 / 2}(J, M ; J-1, M)=2 \boldsymbol{S}^{1 / 2}(J, J-1) \sqrt{\frac{3}{32 \pi} \frac{J^{2}-M^{2}}{J(2 J-1)(2 J+1)}} \sin \theta \\
\boldsymbol{S}_{\theta}^{1 / 2}(J, M ; J-1, M \pm 1)= \pm \boldsymbol{S}^{1 / 2}(J, J-1) \sqrt{\frac{3}{32 \pi} \frac{(J \mp M)(J \mp M-1)}{J(2 J-1)(2 J+1)}} \sqrt{1+\cos ^{2} \theta}
\end{array}\right\}
$$

In our particular problem, observation was made at $\theta=90^{\circ}$, so the angle factor occurring last in eq 10 is unity in all cases. In the following discussion, transverse observation will be assumed, and we shall omit the subscript $\theta=90^{\circ}$. Direct substitution of the line strengths (eq 9), and the state quantum numbers, in eq 10 , gives the values of $\boldsymbol{S}^{1 / 2}$ in table 7 after multiplication by the factor $\sqrt{640 \pi}$ in order to get numbers comparable in magnitude to the estimated intensities. It is noted that the sums of the squares of the values in each of the three boxes of table 7 are proportional to the line strengths $7: 5: 3$.

The unperturbed strength-pattern of table 7 is seen to be radically different from the observed pattern of table 5. Some of the weakest compon- 
ents of table 7, and even the missing component, are among the strongest in table 5. Some of the moderately strong components of table 7 become very weak or are missing observationally. It is these pronounced differences for which the computation of the Paschen-Back perturbation must account. Table 7 furnishes the starting point for this computation, which leads to the perturbed values of $\boldsymbol{S}^{1 / 2}$ initalies in parentheses in table 5 (the sign of $\boldsymbol{S}^{1 / 2}$ is no longer retained in table 5 , since this sign has no observable meaning but is only needed in the computation).

Since the states of ${ }^{5} \mathrm{~S}_{2}$ are unperturbed, we need consider only the effect of the perturbation of the states of ${ }^{5} \mathrm{P}$. Values of $M$ occurring only once in ${ }^{5} \mathrm{P}$ suffer no perturbation ( $M=3$ and -3$)$, so intensities of components from these states are unperturbed. The value 10.95 for $3 \rightarrow 2$ and $-3 \rightarrow-2$ reappears in table 5 as (11.0).

For an $M$ value that appears twice, such as $M=2$, the two components to the same lower state can transfer strength from one to the other. Designating unperturbed strengths by subscript unp and perturbed strengths by subscript $p$, we have from table 7

$$
\boldsymbol{S}_{\text {unp }}(2 \rightarrow 2)=80, \quad \boldsymbol{S}_{\text {unp }}\left(2^{\prime} \rightarrow 2\right)=160,
$$

whereas the perturbed values entered in table 5 , which will be computed presently, are

$$
\boldsymbol{S}_{p}(2 \rightarrow 2)=(14.0)^{2}=196, \quad \boldsymbol{S}_{p}\left(2^{\prime} \rightarrow 2\right)=(6.7)^{2}=44 .
$$

The sum of the strengths of the two components is unchanged by the perturbation, but the weaker has become stronger and the stronger weaker. The change is not always in this direction. If we look at the components $(-2 \rightarrow-2)$ and $\left(-2^{\prime} \rightarrow-2\right)$, which have the same unperturbed strengths as eq 11:

$$
\boldsymbol{S}_{u n p}(-2 \rightarrow-2)=80, \quad \boldsymbol{S}_{u n p}\left(-2^{\prime} \rightarrow-2\right)=160,
$$

the perturbed values of table 5 show the stronger component still stronger, the weaker still weaker:

$$
\begin{aligned}
& \boldsymbol{S}_{p}(-2 \rightarrow-2)=(4.4)^{2}=20, \\
& \boldsymbol{S}_{p}\left(-2^{\prime} \rightarrow-2\right)=(14.8)^{2}=220 .
\end{aligned}
$$

The signs of $\boldsymbol{S}_{\text {unp }}$ and the relative location of the levels are the factors that determine which way the intensity will shift.
The general formulas for making these computations are the following:

Let $A$ be the upper, $B$ the lower of two unperturbed energies, as in eq 2 . Let $\Delta=A-B$ be the unperturbed energy difference $(\Delta>0)$. Let $A_{p}$ be the upper, $B_{p}$ the lower of the two perturbed energies. Let $\Delta_{p}=A_{p}-B_{p}$ be the perturbed energy difference $\left(\Delta_{p}>0\right)$. Then if $\boldsymbol{S}^{3 / 2}(A \rightarrow X)$ and $\boldsymbol{S}^{3 / 2}(B \rightarrow X)$ denote the unperturbed values of $S^{1 / 2}$ from $A$ and $B$ to the same lower state $X$, the strengths of the perturbed components are given by the formulas

$$
\left.\begin{array}{l}
\boldsymbol{S}_{p}^{1 / 2}\left(A_{p} \rightarrow X\right)= \\
\sqrt{\frac{\Delta_{p}+\Delta}{2 \Delta_{p}}} \boldsymbol{S}^{1 / 2}(A \rightarrow X)+\sqrt{\frac{\Delta_{p}-\Delta}{2 \Delta_{p}}} \boldsymbol{S}^{1 / 2}(B \rightarrow X) \\
\boldsymbol{S}_{p}^{1 / 2}\left(B_{p} \rightarrow X\right)= \\
\quad \sqrt{\frac{\Delta_{p}+\Delta}{2 \Delta_{p}}} \boldsymbol{S}^{1 / 2}(B \rightarrow X)-\sqrt{\frac{\Delta_{p}-\Delta}{2 \Delta_{p}}} \boldsymbol{S}^{1 / 2}(A \rightarrow X)
\end{array}\right\}
$$

In our case, for $M=2$, in order to compute eq 13 , we use $A=3.333, B=2.745, \Delta=0.588, A_{p}=$ 3.594, $B_{p}=2.484$, and $\Delta_{p}=1.110$, all in L. U., as given by eq 3 and the discussion preceding eq 3 . Here $A$ and $B$ represent the states 2 and $2^{\prime}$ of ${ }^{5} \mathrm{P}$. If $X$ represents the state $M=2$ of ${ }^{5} \mathrm{~S}$, we need for the right? side of eq 13 the unperturbed values $S^{1 / 2}(2 \rightarrow 2)=8.94, S^{1 / 2}\left(2^{\prime} \rightarrow 2\right)=12.65$, obtained from table 7. Substituting these and the coefficients

$$
\sqrt{\left(\Delta_{p}+\Delta\right) / 2 \Delta_{p}}=0.875, \quad \sqrt{\left(\Delta_{p}-\Delta\right) / 2 \Delta_{p}}=0.485,
$$

on the right of eq 13 we obtain the perturbed values

$$
\left.\begin{array}{l}
S_{p}^{1 / 2}(2 \rightarrow 2)=0.875(8.94)+0.485(12.65)=14.0 \\
S_{p}^{1 / 2}\left(2^{\prime} \rightarrow 1\right)=0.875(12.65)-0.485(8.94)=6.7
\end{array}\right\}
$$

as in eq 12. The same coefficients, 0.875 and 0.485 , are used in eq 13 to obtain the perturbed strengths of components $2 \rightarrow 1$ and $2^{\prime} \rightarrow 1$ :

$\left.\boldsymbol{S}_{p}^{1 / 3}(2 \rightarrow 1)=0.875(-8.94)+0.485(6.32)=-4.8\right)$

$\left.\boldsymbol{S}_{p}^{1 / 2}\left(2^{\prime} \rightarrow 1\right)=0.875(6.32)-0.485(-8.94)=9.9\right\}$

which are entered in table 5 with sign ignored.

From the form of eq 13 we see that when $\boldsymbol{S}^{1 / 2}(A \rightarrow X)$ and $\boldsymbol{S}^{1 / 2}(B \rightarrow X)$ have the same sign, the strength $\boldsymbol{S}^{1 / 2}(A \rightarrow X)$ from the upper state is increased by the perturbation. This is the case in eq 14. On the other hand, when $\boldsymbol{S}^{1 / 2}(A \rightarrow X)$ and 
$\boldsymbol{S}_{3 / 2}(B \rightarrow X)$ have opposite signs, as in eq 15 , the strength from the upper state is ordinarily decreased, but this is not necessarily true.

When there are three or more states of the same $M$ value, e. g., $M=1$, the computation takes a more complex form, of which the above is a special case. As an illustration of the method, let us compute the perturbed intensities $\boldsymbol{S}_{p}^{1 / 3}\left(1^{\prime \prime} \rightarrow 2\right)$, $\boldsymbol{S}_{p}^{1 / 2}\left(1^{\prime \prime} \rightarrow 1\right), \quad \boldsymbol{S}_{p}^{1 / 3}\left(1^{\prime \prime} \rightarrow 0\right)$, which are interesting because their values in table 5 differ so strikingly from the unperturbed values of table 7 .

Using the elements of the determinant eq 5 as coefficients, with the value of $\lambda$ corresponding to $1^{\prime \prime}(\lambda=0.172$ from eq 7$)$, we form the set of homogeneous linear equations

$$
\left.\begin{array}{r}
(1.667-0.172) \alpha+0.596 \beta=0, \\
0.596 \alpha+(0.911-0.172) \beta+0.671 \gamma=0, \\
0.671 \beta+(1.072-0.172) \gamma=0 .
\end{array}\right\}
$$

These equations are to be solved for $\alpha, \beta, \gamma$ subject to the normalization condition $\alpha^{2}+\beta^{2}+\gamma^{2}=1$. The first equation of eq 16 gives $\alpha=-0.399 \beta$; the third gives $\gamma=-0.746 \beta$. Whence $\alpha^{2}+\beta^{2}+\gamma^{2}$ $=(0.159+1+0.557) \beta^{2}=1$, so $\beta=0.763$. It does not matter whether $\beta$ is called + or - , but the relative signs of $\alpha, \beta, \gamma$ are important. Then

$$
\left.\begin{array}{l}
\alpha=-0.304 \\
\beta=+0.763 \\
\gamma=-0.569
\end{array}\right\}
$$

The second of the above homogeneous equations has not been used and will serve as a check

From the way in which eq 16 were formed from eq 4 , it is seen that $\alpha, \beta, \gamma$ are associated respectively with the unperturbed ${ }^{5} \mathrm{P}_{3}^{1},{ }^{5} \mathrm{P}_{2}^{1},{ }^{5} \mathrm{P}_{1}^{1}$, i. e., with the unperturbed states 1, 1', $1^{\prime \prime}$. Quantummechanically, they are the coefficients in the wave function of the perturbed state $1^{\prime \prime}$ when expressed in terms of the unperturbed states $1,1^{\prime}, 1^{\prime \prime}$. The perturbed value of $\boldsymbol{S}_{p}^{1 / 2}\left(1^{\prime \prime} \rightarrow X\right)$ from the perturbed state $1^{\prime \prime}$ to any one of the states $X$ of ${ }^{5} \mathrm{~S}_{2}$ is then given in terms of the unperturbed values

$$
\boldsymbol{S}_{u n p}^{1 / 2}(1 \rightarrow X), \boldsymbol{S}_{u n p}^{1 / 2}\left(1^{\prime} \rightarrow X\right), \boldsymbol{S}_{u n p}^{1 / 2}\left(1^{\prime \prime} \rightarrow X\right)
$$

by using these coefficients in the simple relation

$$
\begin{aligned}
& \boldsymbol{S}_{p}^{1 / 2}\left(1^{\prime \prime} \rightarrow X\right)=\alpha \boldsymbol{S}_{u n p}^{1 / 2}(1 \rightarrow X)+ \\
& \quad \beta \boldsymbol{S}_{u n p}^{1 / 2}\left(1^{\prime} \rightarrow X\right)+\gamma \boldsymbol{S}_{u n p}^{1 / 1 / 2}\left(1^{\prime \prime} \rightarrow X\right) .
\end{aligned}
$$

Thus, using the values of $\alpha, \beta, \gamma$ of eq 17 and the unperturbed values of $\boldsymbol{S}_{u n p}^{1 / 2}$ read from table 6 , we obtain

$$
\begin{aligned}
& \boldsymbol{S}_{p}^{1 / 2}\left(1^{\prime \prime} \rightarrow 2\right)= \\
& \quad-0.304(2.83)+0.763(6.32)-0.569(8.49)=-0.9 \\
& \boldsymbol{S}_{p}^{1 / 2}\left(1^{\prime \prime} \rightarrow 1\right)= \\
& \quad-0.304(11.31)+0.763(6.32)-0.569(-8.49)=6.2 \\
& \boldsymbol{S}_{p}^{1 / 2}\left(1^{\prime \prime} \rightarrow 0\right)= \\
& \quad-0.304(-6.93)+0.763(7.75)-0.569(-3.46)=10.0
\end{aligned}
$$

as entered in table 5. These values are strikingly different from the unperturbed values $8.5,8.5,3.5$, but in striking agreement with the observational estimates 1, 7, 10 .

Two similar computations, on replacing the 0.172 in eq 16 by 1.356 and 2.121 successively (eq 7), will give the perturbed components $1^{\prime} \rightarrow 2,1^{\prime} \rightarrow 1,1^{\prime} \rightarrow 0$, and $1 \rightarrow 2,1 \rightarrow 1,1 \rightarrow 0$, respectively. A check is furnished by the fact that the sum of the strengths of the components that get mixed up must be unchanged by perturbation. Thus, e. g.,

$$
\begin{aligned}
& \boldsymbol{S}_{p}(1 \rightarrow 2)+ \boldsymbol{S}_{p}\left(1^{\prime} \rightarrow 2\right)+\boldsymbol{S}_{p}\left(1^{\prime \prime} \rightarrow 2\right)= \\
& \boldsymbol{S}_{\text {unp }}(1 \rightarrow 2)+\boldsymbol{S}_{\text {unp }}\left(1^{\prime} \rightarrow 2\right)+\boldsymbol{S}_{\text {unp }}(1 \rightarrow 2),
\end{aligned}
$$

or from tables 5 and 7 :

$$
\begin{aligned}
(8.8)^{2}+(6.5)^{2}+ & (0.9)^{2}= \\
& (2.8)^{2}+(6.3)^{2}+(8.5)^{2}, \text { or } 120=120 .
\end{aligned}
$$

Computations similar to the above give the strengths of components from $0,0^{\prime}, 0^{\prime \prime}$ and -1 , $-1^{\prime},-1^{\prime \prime}$.

It should be pointed out that the method of computation sketched in this section needs no alteration whatsoever, except for reversing the arrows, if the combining term ( ${ }^{5} \mathrm{~S}$ in this case) happens to lie above the perturbed term rather than below, as here. The strengths in the case in which both terms have Paschen-Back perturbations would be solved in two stages as follows: Start with the table similar to table 7 enlarged, which gives unperturbed values of $\boldsymbol{S}^{1 / 2}$. Then apply exactly the above procedure to introduce the perturbation into the term on the left of the table, leaving the term on the top in its unperturbed condition, to get a new table. (Remember to keep signs.) As a second stage, introduce the perturbation into the term at the top, working with the new table in which the term at the left is already perturbed, to get a final table in which 
both terms are perturbed. This two-stage procedure is equivalent to multiplication of the original table, considered as a matrix, in front by one matrix and behind by another. This matrix scheme makes it easier to keep track of the arithmetic, but does not involve less or different arithmetic from that in the above procedure.

\section{Weak Paschen-Back Interaction in $\mathrm{N}$ I}

The $g$-values given for the quartet terms of $\mathrm{N}$ I in table 4 were obtained by a procedure similar to that sketched above for $\mathrm{O}$ I. Although the Paschen-Back perturbations in $\mathrm{N}_{\mathrm{I}}$ are much less than those in $\mathrm{O}_{\mathrm{I}}$ (see fig. 1), yet they must be taken into account in determining $g$-values from the experimental data if full accuracy in determining them is to be realized. In the case of $\mathrm{N} \mathrm{I}$, the whole group of Zeeman components arising from transitions between the upper levels $2 p^{2} 3 p{ }^{4} \mathrm{~S}_{11 / 2}^{\circ},{ }^{4} \mathrm{P}_{21 / 2}^{\circ},{ }^{4} \mathrm{P}_{1 \frac{1}{2}}^{\circ},{ }^{4} \mathrm{P}_{01 / 2}^{\circ}$ and the lower levels $2 p^{2} 3 s^{4} \mathrm{P}_{2 \frac{1}{2}},{ }^{4} \mathrm{P}_{1 \frac{1}{2}},{ }^{4} \mathrm{P}_{0 \frac{1}{2}}$ were fitted into a rectangular array from which values of the energies in the magnetic field were derived for all the upper and lower states. The array was similar to table 5 or 6 considerably enlarged. From the energy values, the $g$-values were computed by exactly the procedure sketched above for the oxygen quintets.

We can verify that a weak Paschen-Back interaction in $L S$-coupling will account for the small positional asymmetries and intensity distortions by using the so-called second-order perturbation theory [12, p. 34].

According to this approximate theory, every pair of states of the same $M$ but differing by one unit in $J$ push each other apart by an amount equal to

$$
\epsilon=\frac{\text { square of interaction element (eq 1) }}{\text { distance between states }}=\frac{I^{2}}{\Delta},
$$

all quantities being expressed in the same units. The energy perturbation (eq 18) is to be applied to each of the two states, in the repulsive direction. Where there are more than two interacting states, the perturbations of each pair may be assumed to act independently. Within the accuracy of eq 18, it does not matter whether the perturbed or unperturbed distance is entered in the denominator.
With regard to $\boldsymbol{S}^{1 / 2}$, if $A$ is the upper and $B$ the lower of two interacting states that combine with a state $X$ either above or below, one can show from eq 13 that to the same approximation

$$
\begin{aligned}
& \boldsymbol{S}_{p}^{1 / 2}(A, X)=\boldsymbol{S}_{u n p}^{1 / 2}(A, X)+(I / \Delta) \boldsymbol{S}^{1 / 2}(B, X) \\
& \boldsymbol{S}_{p}^{1 / 2}(B, X)=\boldsymbol{S}_{u n p}^{1 / 6}(B, X)-(I / \Delta) \boldsymbol{S}^{1 / 2}(A, X)
\end{aligned}
$$

where $I$ is the interaction element ( eq 1 ), and $\Delta$ is the distance between states. In the last term, either the perturbed or the unperturbed $\boldsymbol{S}^{1 / 2}$ may be used. Where a state is perturbed by two others, the changes of $\boldsymbol{S}^{1 / 2}$ are additive to this approximation.

Conspicuous among the perturbations of the $\mathrm{N}$ I ${ }^{4} \mathrm{~S} \rightarrow{ }^{4} \mathrm{P}$ of figure 1 are the four intervals of the six strong $\sigma$-components of the center line, which should be equal in the unperturbed case. The observed ${ }^{4}$ wavelengths, intensities, and intervals for the $\sigma$-components of this line, ${ }^{4} \mathrm{~S}_{1 \frac{1}{2}} \rightarrow{ }^{4} \mathrm{P}_{11 / 2}$, are given in the following array together with (at the left) the $M$-values of the transition to which they correspond:

$\begin{array}{rrr}\left(1 \frac{1}{2} \rightarrow \frac{1}{2}\right) & 7437.728 \mathrm{~A}(5) \\ \left(\frac{1}{2} \rightarrow-\frac{1}{2}\right) & 38.306 & (6) \\ \left(-\frac{1}{2} \rightarrow-1 \frac{1}{2}\right) & 38.808 & (4) \\ \left(\frac{1}{2} \rightarrow 1 \frac{1}{2}\right) & 45.907 & (5) \\ \left(-\frac{1}{2} \rightarrow \quad \frac{1}{2}\right) & 46.632 & (6 \\ \left(-1 \frac{1}{2} \rightarrow-\frac{1}{2}\right) & 47.217 & (4)\end{array}$

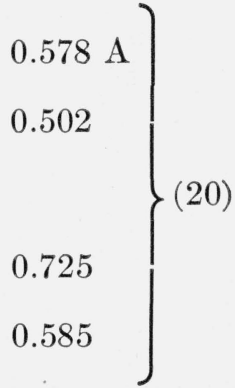

We shall use the above formulas to account for the interval-perturbations and the small, but definite, intensity-distortion exhibited here. As the Lorentz unit, when expressed in angstroms, differs by only 1 percent over the spread of the whole quartet pattern, we shall not change to wave numbers but shall use a mean Lorentz unit of $2.223 \mathrm{~A}$. We shall express the interaction elements (eq 1) in angstrom units and work entirely in angstroms. These interaction elements connecting the states of ${ }^{4} \mathrm{P}$ are

\footnotetext{
${ }_{4}$ The data used in this section for illustrating the approximate theory of weak Paschen-Back interaction are obtained from the one plate reproduced in figure 1. These data do not agree exactly with those given in table 2 , which represent the means of measurement of three plates.
} 


$$
\left.\begin{array}{r}
M= \pm 1 \frac{1}{2}: I=0.490 \mathrm{~L} \cdot \mathrm{U} .=1.09 \mathrm{~A} \\
{ }^{4} \mathrm{P}_{2 \frac{1}{2}},{ }^{4} \mathrm{P}_{1 \frac{1}{2}} \\
M= \pm \frac{1}{2}: I=0.600 \mathrm{~L} \cdot \mathrm{U} .=1.33 \mathrm{~A} \\
{ }^{4} \mathrm{P}_{1 \frac{1}{2}},{ }^{4} \mathrm{P}_{1 / 2} M= \pm \frac{1}{2}: I=0.745 \mathrm{~L} \cdot \mathrm{U} .=1.66 \mathrm{~A}
\end{array}\right\}
$$

The distances between states of ${ }^{4} \mathrm{P}$ needed in the denominator of eq 18 can be obtained with sufficient accuracy by taking the wavelength differences between components coming from the same state of ${ }^{4} \mathrm{~S}$. These distances are

$$
\left.\begin{array}{lcc}
M=1 \frac{1}{2}: & \Delta=25.7 \mathrm{~A} & \\
M=\frac{1}{2}: & 25.9 & \Delta=17.8 \mathrm{~A} \\
M=-\frac{1}{2}: & 26.2 & 19.9 \\
M=-1 \frac{1}{2}: & 26.5 &
\end{array}\right\}
$$

The quartet is erect. The state ${ }^{4} \mathrm{P}_{1 \frac{1}{2}}^{11 / 2}$ has been pushed down by $(1.09)^{2} / 25.7=0.046 \mathrm{~A}$ by ${ }^{4} \mathrm{P}_{2 / 2}^{11 / 2}$ lying above. Similarly ${ }^{4} \mathrm{P}_{11 / 2}^{-1 \frac{1}{2}}$ has been pushed down by $(1.09)^{2} / 26.5=0.045 \mathrm{~A}$. The states ${ }^{4} \mathrm{P}_{1 / 2}^{ \pm 1 / 2}$ have suffered a net upward push, because the ${ }^{4} \mathrm{P}_{1 / 2}$ states below are closer and have larger interaction elements than the ${ }^{4} \mathrm{P}_{21 / 2}$ states above. The net shift upward of ${ }^{4} \mathrm{P}_{1 / 2}^{1 / 2}$ is $(1.66)^{2} / 17.8-(1.33)^{2} / 25.9$ $=0.087 \mathrm{~A}$. The net shift upward of ${ }^{4} \mathrm{P}_{11 / 2}^{-11 / 2}$ is $(1.66)^{2} / 19.9-(1.33)^{2} / 26.2=0.072 \mathrm{~A}$.
When these Paschen-Back perturbations are removed from the wavelengths (eq 20), we find the unperturbed wavelengths and intervals

$$
\begin{gathered}
{ }^{4} \mathrm{~S}_{1 / 2} \rightarrow{ }^{4} \mathrm{P}_{1 \frac{1}{2}} \text { (unperturbed) } \\
\left(1 \frac{1}{2} \rightarrow \quad 1 / 2\right) 7437.728-0.087=7437.641 \\
(1 / 2 \rightarrow-1 / 2) \quad 38.306-0.072=38.234 \\
\left(-1 / 2 \rightarrow-1 \frac{1}{2}\right) \quad 38.808+0.045=38.853 \\
\left(1 / 2 \rightarrow 1 \frac{1}{2}\right) \quad 45.907+0.046=45.953 \\
(-1 / 2 \rightarrow \quad 1 / 2) \quad 46.632-0.087=46.545 \\
\left(-1 \frac{1 / 2}{1} \rightarrow-1 / 2\right) \quad 47.217-0.072=47.145
\end{gathered}
$$

\begin{tabular}{|c|c|c|c|c|c|}
\hline & \multicolumn{4}{|c|}{${ }^{4} \mathrm{~S}_{11 / 2}$} \\
\hline & & $M=11 / 2$ & $M=1 / 2$ & & $M=-1 \frac{1}{3}$, \\
\hline \multirow{6}{*}{${ }^{4} \mathrm{P}_{21 / 2}$} & $M=2 \frac{1}{2}$ & -10 & & & \\
\hline & $1 \frac{1}{2}$ & +11 & -6 & & \\
\hline & $1 / 2$ & +2 & +12 & -3 & \\
\hline & $-1 / 2$ & & +4 & +12 & -1 \\
\hline & $-1 \frac{1}{2}$ & & & +7 & +10 \\
\hline & $-2 \frac{1}{2}$ & & & & +10 \\
\hline \multirow{4}{*}{${ }^{4} \mathrm{P}_{11 / 2}$} & $M=1 \frac{1}{2}$ & +11 & +5 & & \\
\hline & $1 / 2$ & +5 & +0 & +6 & \\
\hline & $-1 / 2$ & & +6 & -2 & +4 \\
\hline & $-1 \frac{1}{2}$ & & & +4 & -12 \\
\hline \multirow[t]{2}{*}{${ }^{4} \mathrm{P}_{3 / 2}$} & $M=1 / 2$ & +6 & $-6^{1 / 2}$ & -3 & \\
\hline & $-1 / 2$ & & +2 & -6 & -7 \\
\hline
\end{tabular}

The unequal intervals of eq 20 are seen to become quite equal within observational error when the Paschen-Back perturbation is removed. The asymmetries of position of the rest of the patterns are similarly accounted for by this theory.

In an unperturbed pattern, the six components of eq 20 should have values of $\boldsymbol{S}^{1 / 2}$ approximately in the ratio $5: 6: 5: 5: 6: 5$ (from 10), with four components of equal intensity. We attempt to account for the inequality of these four components, as well as for the intensity-asymmetries in the balance of the pattern. The observed estimated intensities, to which have been attached the proper signs for the unperturbed $\boldsymbol{S}^{1 / 2}$ taken from eq 8 and eq 10, are

\section{Zeaman Effect and $\boldsymbol{g}$-Values}


We shall compute the last (perturbation) terms of eq 19 for each of these components and subtract them from the observed values to see if we regain a symmetrical pattern. In doing so we shall use for the $\boldsymbol{S}^{3 / 2}$ of the perturbing component in the last term of eq 19 just the observed intensity estimate of this component. Thus for ${ }^{4} \mathrm{~S}_{11 / 2}^{1 / 2} \rightarrow{ }^{4} \mathrm{P}_{1}^{11 / 3}$ the perturbation is by a state lying higher, so we use eq $19 \mathrm{~b}$, substituting from eq 21, 22, and 24 to get

$$
+5=\boldsymbol{S}_{p}^{1 / 2}=\boldsymbol{S}_{u n p}^{1 / 6}-(1.09 / 25.7)(-6),
$$

from which $\boldsymbol{S}_{u n p}^{1 / 2}=4.7$. When we consider ${ }^{4} \mathrm{~S}_{11 / 2}^{-11 / 2} \rightarrow$
${ }^{4} \mathrm{P}_{11 / 2}^{-1 / 2}$, which should have equal unperturbed intensity, there is perturbation both from above and below. We use the correction term given by eq $19 \mathrm{~b}$ for perturbation by the state above and that given by eq 19a for perturbation by the state below. Thus for this component,

$+4=\boldsymbol{S}_{p}^{1 / 2}=\boldsymbol{S}_{u n p}^{1 / 2}-(1.33 / 26 / 2)(-1)+(1.66 / 19.9)(-7)$

from which $\boldsymbol{S}_{u n p}^{1 / 2}=4.5$. In a similar way all the unperturbed values of $\boldsymbol{S}^{1 / 2}$ are computed, and are collected in the following table:

\begin{tabular}{|c|c|c|c|c|c|}
\hline & \multicolumn{4}{|c|}{${ }^{4} \mathrm{~S}_{13 / 2}$} \\
\hline & & $M=1 \frac{1}{2}$ & $M=1 / 2$ & $M=-1 / 2$ & $M=-11 / 2$ \\
\hline \multirow{6}{*}{${ }^{4} \mathrm{P}_{21 / 2}$} & $M=2 \frac{1}{2}$ & -10 & & & \\
\hline & $11 / 2$ & 10.5 & -6.2 & & \\
\hline & $1 / 2$ & 1. 7 & 12.0 & -3.3 & \\
\hline & $-1 / 2$ & & 3. 7 & 12.1 & -1.2 \\
\hline & $-1 \frac{1}{2}$ & & & 6. 8 & 10.5 \\
\hline & $-2 \frac{1}{2}$ & & & & 10 \\
\hline \multirow{4}{*}{${ }^{4} \mathrm{P}_{11 / 2}$} & $M=1 \frac{1}{2}$ & 11.5 & 4. 7 & & \\
\hline & $1 / 2$ & 4. 5 & 1. 3 & 6. 1 & \\
\hline & $-1 / 2$ & & 5. 9 & -0.9 & 4. 5 \\
\hline & $-1 \frac{1}{2}$ & & & 4. 3 & -11.6 \\
\hline \multirow[t]{2}{*}{${ }^{4} \mathrm{P}_{3 / 2}$} & $M=1 / 2$ & 6.5 & -6.5 & -2.4 & \\
\hline & $-1 / 2$ & & 2. 5 & -6.2 & -6.7 \\
\hline
\end{tabular}

Comparison of eq 25 with eq 24 shows that in all cases the intensity-perturbation is in the right direction and of the right order of magnitude to account for the observed asymmetries. This is a definite check on the adequacy of this theory in connection with these intensity estimates, because particular care was taken in the estimates to note which of the pair of almost symmetrical components was the stronger. In the three cases where there was no detectable difference in the strength of such a pair, the computed PaschenBack perturbation is seen to be 0.1 or less on this scale.

\section{Summary}

Spectrograms of the Zeeman patterns of the red and infrared lines of various metals show the patterns of atmospheric nitrogen and oxygen lines as well. The nitrogen multiplets result from the term combinations $3 s^{4} \mathrm{P}-3 p^{4} \mathrm{D}^{\circ}$, $3 s^{4} \mathrm{P}-3 p{ }^{4} \mathrm{P}^{\circ}, 3 s^{4} \mathrm{P}-3 p{ }^{4} \mathrm{~S}^{\circ}$, and $3 s^{2} \mathrm{P}-3 p^{2} \mathrm{P}^{\circ}$, while those of oxygen result from the combinations $3 s^{5} \mathrm{~S}^{\circ}-3 p{ }^{5} \mathrm{P}$ and $3 s^{3} \mathrm{~S}^{\circ}-3 p^{3} \mathrm{P}$. On spectrograms made at the National Bureau of Standards, with magnetic field strengths of 35,000 gausses, and on Massachusetts Institute of 
Technology spectrograms, with fields in excess of 85,000 gausses, the nitrogen and oxygen patterns exhibit various degrees of distortion, both in the positions and in the intensities of the magnetic components. The nitrogen patterns exhibit only slight distortion or none at all. With the oxygen patterns the case is different: the quintet group, at 7771 to $7775 \mathrm{~A}$, shows marked distortion at two different fields and bears no resemblance to either a weak-field pattern or to a Lorentz triplet; the triplet group, at $8446 \mathrm{~A}$, however, shows a nearly perfect Lorentz triplet pattern with very weak $\pi$-satellites at the normal triplet separation. The interpretation of these patterns has afforded an interesting application of quantum theory to the elucidation of the Paschen-Back effect. The $g$-values that we have derived for the energy levels of $\mathrm{NI}_{\mathrm{I}}$ and $\mathrm{O}_{\mathrm{I}}$ are the first to be announced for neutral atoms of atomic number less than 10 , neon, and are found to conform, within observational error, with those required for $L S$-coupling, despite the fact that the term intervals, except those of $3 p{ }^{4} \mathrm{D}^{\circ}$ of $\mathrm{NI}$, do not conform with the Landé ratios.
We thank Professor G. R. Harrison for placing at our disposal the MIT spectrograms, on which a large share of this investigation is based.

\section{References}

[1] F. Paschen and E. Back, Ann. phys. [4] 39, 897 (1912) [4] 40, 960 (1913).

[2] C. Mihul, Ann. phys. [10] 9, 261 (1938).

[3] J. B. Green and H. N. Maxwell, Phys. Rev. 51, 243 (1937).

[4] G. R. Harrison and F. Bitter, Phys. Rev. 57, 15 (1940).

[5] R. W. Wood, Nature 140, 723 (1937).

[6] E. Back, Ann. Physik. [4] \%o, 333 (1923).

[7] W. F. Meggers and K. Burns, BS Sci. Pap. 18, 191 (1922) S441.

[8] B. Edlén, Bergstrand's Festskrift, p. 135 (1938).

[9] W. F. Meggers, Pub. Allegheny Observatory 6, 13 (1919).

[10] C. E. St. John, et al., Carnegie Inst. of Washington, Pub. No. 396 (1928).

[11] H. N. Russell, Proc. Nat. Acad. Sci. 11, 322 (1925).

[12] E. U. Condon and G. Shortley, Theory of atomic spectra (Cambridge University Press, 1935).

[13] R. T. Birge, Reports on progress in physics, VIII, p. 131 (London, 1941).

Washington, March 1, 1948. 